\title{
The OncoLoop Network-based Precision Cancer Medicine Framework
}

\author{
Alessandro Vasciaveo ${ }^{1, \dagger}$, Min Zou ${ }^{2, \dagger}$, Juan Martín Arriaga2,†, Francisca Nunes de \\ Almeida ${ }^{2, \dagger \dagger}$, Eugene F. Douglass, $\mathrm{Jr}^{1, \dagger^{1 \dagger}}$, Maho Shibata ${ }^{3,4,5}$, Antonio Rodriguez-Calero6,7, Simone

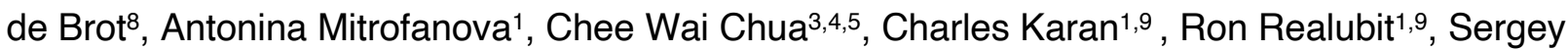 \\ Pampou ${ }^{1,9}$, Jaime Y. Kim², Eva Corey ${ }^{10}$, Mariano Alvarez ${ }^{1,11}$, Mark A. Rubin ${ }^{12,13}$, \\ Michael M. Shen ${ }^{1,3,4,5,14}$, Andrea Califano1,3,9,14,15,16, and Cory Abate-Shen ${ }^{1,2,3,5,14,17}$
}

\section{Affiliations}

1 Department of Systems Biology, Columbia University Irving Medical Center, New York, USA 10032

2 Department of Molecular Pharmacology and Therapeutics, Columbia University Irving Medical Center, New York, NY USA 10032

3 Department of Medicine, Columbia University Irving Medical Center, New York, USA 10032

4 Department of Genetics and Development, Columbia University Irving Medical Center, New York, NY USA 10032

5 Department of Urology, Columbia University Irving Medical Center, New York, NY USA 10032

6 Department of Biomedical Research, University of Bern, Bern, Switzerland 3008

7 Institute of Pathology, University of Bern and Inselspital, Bern, Switzerland 3008

8 COMPATH, Institute of Animal Pathology, University of Bern, Switzerland 3012

9 J.P. Sulzberger Columbia Genome Center, Columbia University Irving Medical Center, New York, NY USA 10032

10 Department of Urology, University of Washington, Seattle, WA USA 98195

11 DarwinHealth Inc, New York, NY

12 Department of Biomedical Research, University of Bern, 3008, Bern, Switzerland.

13 Bern Center for Precision Medicine (BCPM) Bern, Switzerland 3008

14 Herbert Irving Comprehensive Cancer Center, Columbia University Irving Medical Center, New York, USA 10032

15 Department of Biochemistry \& Molecular Biophysics, Columbia University Irving Medical Center, New York, USA 10032

16 Department of Biomedical Informatics, Columbia University Irving Medical Center, New York, USA 10032

17 Department of Pathology and Cell Biology, Columbia University Irving Medical Center, New York, NY USA 10032

$\dagger$ These authors contributed equally

t† These authors contributed equally

Correspondence to: Andrea Califano (ac2248@cumc.columbia.edu) 


\section{Current Addresses:}

Min Zou

Arvinas

5 Science Park

New Haven, CT 06511

Juan M. Arriaga

Department of Oncological Sciences and Department of Urology Icahn School of Medicine at Mount Sinai

One Gustave L. Levy Place

New York, NY 10029

Maho Shibata

Department of Anatomy \& Cell Biology and GW Cancer Center

The George Washington University School of Medicine \& Health Sciences

$80022^{\text {nd }}$ Street NW, Suite 8050

Washington, DC 20052, USA

Antonina Mitrofanova

Department of Health Informatics

Rutgers School of Health Professions

Rutgers Biomedical and Health Sciences, Newark, NJ 07107, USA

Chee Wai Chua

State Key Laboratory of Oncogenes and Related Genes

Renji-Med X Clinical Stem Cell Research Center

Department of Urology

Ren Ji Hospital, School of Medicine, Shanghai Jiao Tong University

160 Pujian Road

15th Floor, Building No. 17

Pudong New District

Shanghai 200127, China

Ronald Realubit

Regeneron Pharmaceuticals, Inc.

777 Old Saw Mill River Rd.

Tarrytown, NY 10591

Eugene F. Douglass, Jr

Department of Pharmaceutical \& Biomedical Sciences

College of Pharmacy

University of Georgia

Room 353

RC Wilson Pharmacy, Athens, GA 30602 


\section{Summary}

Prioritizing cancer treatment at the individual patient level remains challenging and performing coclinical studies using patient-derived models in real-time is often unfeasible. To circumvent these challenges, we introduce OncoLoop, a precision medicine framework to predict drug sensitivity in both a human tumor and its highest-fidelity (cognate) model(s) - for contextual in vivo validationby leveraging perturbational profiles of clinically-relevant oncology drugs. As proof-of-concept, we applied OncoLoop to prostate cancer using a series of genetically engineered mouse models (GEMMs) that capture the broad spectrum of disease states, including metastatic, castrationresistant, and neuroendocrine prostate cancer. Interrogation of published cohorts revealed that most patients were represented by at least one cognate GEMM-derived tumor (GEMM-DT), based on Master Regulator (MR) conservation analysis. Drugs recurrently predicted to invert MR protein activity in patients and their cognate GEMM-DTs were successfully validated, including in two cognate allografts and one patient derived xenograft (PDX). OncoLoop is highly generalizable and can be extended to other cancers and other pathologies. 
Vasciaveo et al.

\section{Introduction}

Systematic prediction of drug sensitivity in vivo remains a major challenge in translational biology for most cancer types. This is due, at least in part, to the inherent heterogeneity of tumors, which makes it difficult to optimize treatments on an individual patient basis. It is further compounded by difficulties in establishing high-fidelity, patient-derived models that recapitulate the biology, complexity, and heterogeneity of an individual patient's tumor-including interaction with a competent immune system - for co-clinical validation. Indeed, depending on tumor type, establishment of patient-derived xenograft (PDX) models can take several months to more than a year (Mattar et al., 2018; Tentler et al., 2012), thus compromising the ability to predict and validate drug efficacy in a time-frame that is compatible with patient treatment, especially in the aggressive metastatic setting. Moreover, PDX models lack a competent immune system, thus affecting drug activity assessment accuracy in vivo. They are also prohibitively low yield in some cancers, thus preventing widespread utilization. Patient-derived organoid models have become increasingly more accessible and representative; however, these are also limited in their ability to model the stromal and immune microenvironment (Bleijs et al., 2019; Schutgens and Clevers, 2020). Finally, although human cell lines derived from patient tumors or metastases are widely available for many cancers, they rarely represent the full spectrum of cancer phenotypes observed in patients and present notoriously idiosyncratic dependencies, as a result of the myriad of genetic and epigenetic alterations they accrue to survive in vitro. In principle, geneticallyengineered mouse models (GEMMs), which are now widely available for many cancer types (Kersten et al., 2017), can provide valuable resources for studying drug response in the whole organism in the context of the native milieu in which tumors arise, and in the presence of an intact immune system. However, methods to assess the fidelity of GEMMs to a human tumor - in terms 
of recapitulating its biology and drug sensitivity for effective use in co-clinical studies-are still lacking.

These general challenges are exemplified in prostate cancer $(\mathrm{PCa})$, which remains the most prevalent form of cancer in men and a leading cause of cancer-related death (Siegel et al., 2021). PCa is characterized by its wide range of disease outcomes; in particular, while most patients typically present with locally-invasive disease that has a 5-year survival of $>90 \%$, advanced PCa has a 5-year survival of $<30 \%$. The first line treatment for patients with advanced PCa is androgen deprivation therapy (ADT), which initially leads to tumor regression but ultimately to the emergence of castration-resistant prostate cancer (CRPC) (Sartor and de Bono, 2018; Scher and Sawyers, 2005; Watson et al., 2015). Notably, CRPC is often metastatic (mCRPC) and associated with highly aggressive disease variants, including neuroendocrine prostate cancer (NEPC) (Rickman et al., 2017; Scher and Sawyers, 2005; Watson et al., 2015). However, the establishment of patient-derived xenograft (PDX) as well as patient-derived organoid models has been particularly low yield for PCa, especially for the advanced cancer phenotypes (Gao et al., 2014; Nguyen et al., 2017; Puca et al., 2018).

Although for many decades the standard of care for patients with advanced PCa was limited to taxane-based chemotherapy, the clinical landscape has greatly expanded in recent years. Nowadays, the current standard of care for advanced PCa is based on second generation androgen signaling inhibitors, such as Enzalutamide and Abiraterone, which are initially effective but often associated with treatment failure (Sartor and de Bono, 2018). Promising new agents include immunotherapeutics, such as Sipuleucel-T and PD-1 inhibitors, radionuclide therapy, such as radium-223, and various therapies targeting pro-tumorigenic pathways, including poly adenosine diphosphate-ribose polymerase (PARP) inhibitors (Crawford et al., 2015; Logothetis et al., 2018; Nuhn et al., 2019; Sartor and de Bono, 2018). However, several features of PCa, 
Vasciaveo et al.

including its inherent molecular heterogeneity, the infrequency of driver mutations, and long oncogenic tail (Armenia et al., 2018), make it difficult to determine which specific treatments may be most effective for specific patients. Consequently, pursuing the broad range of targeted therapies available today against specific drivers or pro-tumorigenic pathways in $\mathrm{PCa}$ is challenging, since it is difficult to determine which patients would benefit from specific treatment.

To overcome these inherent challenges and to optimally leverage the ever-increasing repertoire of cancer models, we developed OncoLoop, an integrated computational/experimental framework designed to first identify high-fidelity models for a given human tumor (cognate model, hereafter) and then to use RNA-sequencing (RNA-seq) profiles from both the patient and its cognate model's tumor to predict optimal drug treatments, based on large-scale drug perturbation profiles, for preclinical evaluation in the latter. More specifically, the cognate models of a given patient are identified by assessing patient vs. model Master Regulator (MR) protein activity conservation (Alvarez et al., 2016; Alvarez et al., 2019), while drugs are prioritized based on their ability to invert the MR-activity signature of both a patient tumor and its cognate model (Alvarez et al., 2018).

Supporting this strategy, we have shown that MR proteins represent mechanistic determinants of tumor transcriptional state and that inversion of their activity effectively abrogates tumor viability (Alvarez et al., 2018; Aytes et al., 2014; Paull et al., 2021). MR-activity signature inversion is effectively assessed experimentally by leveraging large-scale RNA-seq profiles of selected cell lines, perturbed with a library of FDA approved and late stage (i.e., phase II/III trials) experimental oncology drugs as well as vehicle control (Alvarez et al., 2018). To generate drug perturbation profiles that recapitulate patient-relevant drug mechanism of action (MoA), cell lines are pre-selected based on their ability to recapitulate the MR-activity signature of a majority of human tumors in publicly available cohorts (Douglass et al., 2022). Thus, OncoLoop identifies the 
Vasciaveo et al.

most statistically significant three-way relationships between a tumor, its highest-fidelity cognate model(s), and one or more drugs predicted to invert their MR-activity signature (hereafter, MRinverter drugs).

As proof-of-concept, we applied this framework to $\mathrm{PCa}$, for which both large-scale human patient cohorts-comprising both primary tumors and metastases (Abida et al., 2019; Cancer Genome Atlas Research, 2015) - and an extensive repertoire of GEMMs ((Arriaga and AbateShen, 2019) and this report) are available. These analyses revealed that a majority of patients in these cohorts had at least one cognate GEMM-Derived Tumor (GEMM-DT). To identify MRinverter drugs, we leveraged PanACEA (Douglass et al., 2022), a large collection of drug perturbational profiles in cell lines matched to cohorts representing 23 tumor subtypes, including PCa. Three out of four predicted drugs induced highly significant growth inhibition of tumor allografts from the cognate GEMM-DT. Furthermore, to support the translational relevance of the approach in a co-clinical context, we validated two of the four drugs also predicted as MR-inverters for a cognate PCa PDX model, which resulted in complete abrogation of tumor growth. Taken together our findings show that OncoLoop provides an effective framework for the rapid identification and evaluation of patient-relevant drugs in pre-existing cognate models thus supporting its co-clinical application. 
Vasciaveo et al.

\section{Results}

Conceptual overview of OncoLoop: We developed OncoLoop with the purpose of identifying drugs poised to benefit patients, whose response could be evaluated in co-clinical models, using transcriptomic data (i.e., RNA-seq profiles), see conceptual framework in Fig. 1A. The analysis leverages regulatory networks - reverse-engineered from large, tumor-specific RNA-seq profile datasets - to first identify cognate GEMM-DTs, based on MR-activity signature conservation with a human tumor, and then to prioritize candidate MR-inverter drugs for both the human tumor and the cognate GEMM-DT, based on drug perturbation profiles of tumor-related cell lines. To demonstrate feasibility of the OncoLoop framework, we focused on PCa, for which we have generated a large series of GEMMs; for other cancer types, however, PDX models may be used if large, representative collections are available.

Data generation: We leveraged large scale RNA-seq profiles from: (i) a comprehensive series of GEMMs representing a broad spectrum of PCa phenotypes; (ii) publicly-available RNA-seq profiles from primary tumors in The Cancer Genome Atlas (TCGA) (Cancer Genome Atlas Research, 2015) and metastases in the Stand Up to Cancer consortium-Prostate Cancer Foundation CRPC500 cohort (SU2C) (Abida et al., 2019); (iii) large-scale drug perturbation profiles from tumor-related cell lines (Douglass et al., 2022); and (iv) RNA-seq profiles from wellcharacterized human PCa PDX models (Nguyen et al., 2017) (Fig. 1B).

Protein activity analysis: OncoLoop requires accurate protein activity assessment for the assessment of MR-activity signatures, identification of cognate models of human tumors, and prediction of MR-inverter drugs. This is accomplished using the VIPER algorithm (Alvarez et al., 2016), which transforms RNA-seq profiles into accurate protein activity profiles, as recently validated by antibody-based protein abundance measurements (Obradovic et al., 2021). Akin to 
a highly multiplexed gene reporter assay, VIPER uses the expression of a protein's tissue-specific targets (regulon) to measure its activity. The repertoire of targets of all regulatory and signaling proteins in a specific tissue context (i.e., context-specific interactome) is generated by analyzing large-scale, tissue-specific RNA-seq profiles using the ARACNe algorithm (Basso et al., 2005). To support accurate, model-specific protein activity measurements, we reverse-engineered separate regulatory networks from large-scale patient-, GEMM-, and PDX-specific RNA-seq datasets (Fig. 1B).

GEMM cohort characterization and cognate model identification: First, we analyzed VIPER-based protein activity profiles from available GEMM-DTs to identify molecularly distinct subtypes and to demonstrate their relevance to the disease spectrum of human PCa. Next, we identified cognate GEMM-DTs for human primary PCa tumors in the TCGA cohort and metastases in the SU2C cohort, based on MR-activity signature conservation (Fig. 1C). This revealed broad coverage, with $78 \%$ and $93 \%$ of TCGA and SU2C tumors, respectively, matched to at least one cognate GEMM-DT.

Assessing drug mechanism of action (MoA) in MR-matched cell lines: To predict drug sensitivity, we leveraged human tumor-relevant drug perturbation profiles generated for two PCa cell linesthe androgen dependent LNCaP and the metastatic, androgen-independent DU145 cell lines. These were selected because they jointly provided high-fidelity models for $>80 \%$ of the tumors in the TCGA cohort, based on MR-activity signature conservation, see STAR Methods. To focus on the more aggressive mCRPC patients, we relied on RNA-seq profiles of DU145 cells treated with a library of FDA-approved and late-stage experimental oncology drugs and vehicle control (DMSO) (i.e., Drug Perturbation Profiles). Finally, the proteome-wide MoA of each drug was assessed by using VIPER to measure the differential protein activity in drug-treated versus 
Vasciaveo et al.

control-treated cells and was used to identify optimal MR-inverter drugs for patient- and cognate GEMM-DT pairs (Fig. 1C).

Closing the Loop: Having "matched" each patient tumor $(P)$ to a cognate GEMM-DT $(G)$ and assessed each drug $(D)$ as a potential MR-inverter for both tumors, we ranked all $(P, G, D)$ triplets (PGD-loops hereafter), based on the integration of three distinct $z$-scores, assessing the statistical significance of (a) the similarity of a patient- and GEMM-DT MR-activity signature, (b) the drug's MR-activity inversion as predicted from the patient tumor, and (c) the drug's MR-activity inversion as predicted from the cognate GEMM-DT, respectively (Fig 1C,D).

In vivo validation: To identify candidates with potential relevance to clinical practice, we focused on clinically available drugs that were most frequently nominated by the analysis of the human tumor cohorts. Among these, we evaluated the ability of the four top-predicted ones to inhibit tumor growth and to recapitulate the predicted MR-activity inversion in vivo, via preclinical studies in allografts and PDX models (Fig. 1D). The following sections discuss each of these steps in detail.

A GEMM resource that models PCa progression: An essential requirement for OncoLoop analysis is the availability of pre-existing high-fidelity models-that accurately represent the complexity and dependencies of the corresponding human tumors. This enables co-clinical validation of predicted drugs within a time-frame relevant to the patient's care or preclinical validation of candidate drugs for follow-up clinical trials. Toward this end, we assembled an extensive series of GEMMs that recapitulate the complexity of human PCa phenotypes, and model common genetic and/or pathway alterations that are prevalent in human PCa (Fig. 2A-E, Fig. S1A-C; Table S1A,B). The phenotypes of some of these GEMMs have been described previously (Arriaga et al., 2020; Aytes et al., 2013; Floc'h et al., 2012; Wang et al., 2012; Zou et 
Vasciaveo et al.

al., 2017). A complete list of GEMMs used in this study is provided in Table S1A,B; the entire GEMM series is available from the Jackson Laboratory (see Table S1).

These GEMMs are based on the Nkx3.1 CreERT2 allele (Fig. 2A) (Wang et al., 2009), which simultaneously introduces a prostate-specific inducible Cre driver and is heterozygous null for the Nkx3.1 homeobox gene, as is common in early stage human PCa (Fig. S1A) (Cancer Genome Atlas Research, 2015). We generated the baseline NP mice (for $\underline{N} k x 3.1^{\text {CreERT2/+; }}$ Pten ${ }^{\text {floxfllox }}$ mice) by crossing the Nkx3.1 ${ }^{\text {CreERT2 }}$ allele with a Pten conditional allele (Fig. 2A) (Lesche et al., 2002), since PTEN mutations are prevalent from the earliest to most advanced stages of human PCa (Fig. S1A) (Abida et al., 2019; Cancer Genome Atlas Research, 2015). The NP mice were then crossed with various other alleles to model: (i) up-regulation of Erg in the NPE mice (E for Rosa26ERG/ERG; (Chen et al., 2013)); (ii) a missense mutation of Tp53 in the NPp53mut mice (for Trp53LSL-R27OH/flox; (Olive et al., 2004)); (iii) up-regulation of $c-M y c$ in the NPM mice (M for Hi-Myc, (Ellwood-Yen et al., 2003)); (iv) an activating mutation of Braf in the NPB mice (B for B-RafV6ooE; (Dankort et al., 2007); and (v) an activating mutation of Kras in the NPK mice (K for Kras ${ }^{\text {LLL-G12D; }}$ (Jackson et al., 2001)) (Fig. 2A). These GEMMs also incorporate a conditionally-activatable fluorescent reporter, the Rosa26-CAG-LSL-EYFP allele (Madisen et al., 2010), for high-efficiency lineage marking of tumors and metastases (Arriaga et al., 2020; Zou et al., 2017).

Since tumor induction is based on an inducible Cre, expression of the relevant alleles following Cre-mediated gene recombination is not dependent on androgens, with the exception of the Hi-Myc allele, which is under the control of a constitutive Probasin promoter (Ellwood-Yen et al., 2003). Consequently, we could analyze tumor progression in both castration-sensitive and castration-resistant contexts (Fig. 2B-E, Fig. S2A). In particular, as evident by MRI imaging of NPp53 mice, surgical castration leads to initial tumor regression followed by eventual outgrowth 
Vasciaveo et al.

of castration-resistant tumors (Fig. 2B); therefore, this GEMM series recapitulates both hormonesensitive and castration-resistant PCa.

This GEMM cohort could be subdivided into three outcome groups $\left(O G_{1}-O G_{3}\right)$, based on overall survival and associated hormone-sensitive and castration-resistant PCa phenotypes (Fig. 2B-E; Fig. S2A,B; Table $S 1 A, B$ ). Those in $O G_{1}$, which include the NP and NPE mice, developed indolent, non-lethal and non-metastatic tumors with mostly benign, PIN-like histology and low levels of proliferation, as evident by Ki67 staining. Those in $O G_{2}$, including the $N P p 53^{m u t}$ and NPM mice, were characterized by lethality within one year, were prone to develop metastasis, and displayed highly heterogeneous and proliferative histopathological phenotypes. Finally, those in $O G_{3}$, including the $N P B$ and $N P K$ mice, were characterized by lethality by 6 months of age, which was accompanied by highly penetrant metastasis and high-grade poorly differentiated histopathology.

To facilitate preclinical investigations, we established allograft and organoid models from representative GEMMs (Fig. S3A,B, Table S1A). Allografts were generated by implanting freshly collected primary tumors into the flank of nude mouse hosts and were passaged at least twice prior to analysis. Organoids were generated by FACS sorting to isolate the lineage-marked primary tumor cells, which were then cultured in vitro for up to five passages. The histopathology of the resulting allograft and organoid models were similar to the parental tumors from which they were derived (Fig. S3A,B). Interestingly, while we were able to generate organoids from GEMMs in each outcome group $\left(O G_{1}-O G_{3}\right)$, we were only able to generate allograft models from the $O G_{2}$ and $O G_{3}$ tumors, but not from indolent $O G_{1}$ tumors. Thus, we have generated an extensive resource of PCa GEMMs, as well as established culturable and transplantable models of these GEMMs. In the current study, we performed in vivo preclinical analyses and therefore used the 
Vasciaveo et al.

allograft rather organoid models; however, we envision that the use of organoid models for in vitro validation will be beneficial for future investigations.

GEMM subtypes recapitulate human PCa phenotypes: To characterize the molecular features of the GEMM cohort, we generated RNA-seq profiles from benign prostate tissue and prostate tumors from 136 individual mice. First, we reverse engineered a GEMM-specific ARACNe interactome from these RNA-seq profiles (Table S2A) (Basso et al., 2005). The resulting interactome supersedes a previously published mouse interactome that was assembled from Illumina gene expression microarrays of a less comprehensive GEMM cohort (Aytes et al., 2014). Not surprisingly, the new RNA-seq-based interactome outperformed the microarray-based interactome. For example, bioactivity analysis-which assesses the ability of an interactome to recapitulate differential protein activity across distinct phenotypes (see STAR Methods) - showed that differentially active proteins had significantly higher average normalized enrichment score $\mu_{\mathrm{NES}, \mathrm{New}}=3.79$ in the new interactome, vs. $\mu_{\mathrm{NES}, \mathrm{Old}}=1.96$ in the previous one $\left(P=2.2 \times 10^{-16}\right.$, by 2-sided Kolmogorov-Smirnov test; Fig. S4A). For instance, while the androgen receptor (AR) regulon-a critical determinant of prostate differentiation and tumorigenesis (Shen and AbateShen, 2010) - was a poor predictor of AR activity in the previous interactome (Aytes et al., 2014), it is highly predictive in the new one (see Fig. 3A, S4B,C).

To define GEMM molecular subtypes and to assess their relationship to human PCa, we focused on a subset of 91 GEMM-DTs selected as having the most physiologically-relevant histopathological phenotypes (Table S1B). We first transformed their transcriptional profiles to VIPER-measured protein activity profiles using the GEMM PCa interactome, and then performed protein activity-based cluster analysis (Fig. 3A; Table S2B,C), see STAR Methods. To generate the differential gene expression signatures necessary for VIPER analysis, we compared each sample to the average of all 91 samples, thus helping identify proteins with the greatest differential 
Vasciaveo et al.

activity across all GEMM-DTs. Protein activity-based cluster analysis, which significantly outperforms gene expression-based clustering (Paull et al., 2021) identified 5 clusters, corresponding to 5 molecularly distinct subtypes $\left(C_{1}-C_{5}\right)$ associated with disease aggressiveness with respect to outcome and metastasis (Fig. 3A; Fig. S4B; Table S2C), see STAR Methods. In particular, subtypes $C_{1}$ and $C_{2}$ mainly comprise tumors from the indolent outcome group $\left(O G_{1}\right)$, while subtypes $C_{3}-C_{5}$ comprise tumors from the two more aggressive outcome groups $\left(O G_{2}\right.$ and $\left.O G_{3}\right)$. Interestingly, the more indolent subtype $C_{1}$ is mostly comprised of castration-resistant tumors while $C_{2}$ includes mostly hormone-sensitive tumors. Furthermore, $>90 \%$ of the tumors in $C_{4}$ and $C_{5}$ had progressed to metastases, compared to only $50 \%$ of those in $C_{3}$, and $11 \%$ of those in $C_{1}$ and $C_{2}$ (Fig. 3A; Fig. S4B).

With respect to their molecular phenotypes, $C_{1}-C_{5}$ tumors were characterized by aberrant activity of a novel protein set that effectively distinguished each subtype (Fig. 3A). These include homeodomain proteins (e.g., Cdx1, Pdx1, Nkx2.6, Nkx3.1, Pitx1) and other transcriptional regulatory proteins (e.g., Gata5, Sox15, Sox7) that have established roles in cellular differentiation and cell lineage control in other tissue contexts. The coordinated switch between the molecular programs regulated by the top MR protein activities of each subtype was evident by the striking differential expression of the transcriptional targets of the most differentially active MRs of each subtype (Fig. 3B, Fig. S5). While the most significantly active MRs in subtypes $C_{1}-C_{5}$ are novel, differential activity of proteins with an established role in human and mouse PCa progression was clearly evident (Fig. 3A; Fig. S4C). In particular, the indolent subtypes $C_{1}$ and $C_{2}$ present high activity of Nkx3.1, p63, and Ar, which are associated with well-differentiated PCa (Shen and Abate-Shen, 2010). In contrast, the most aggressive and highly metastatic subtypes $C_{4}$ and $C_{5}$, present high activity of FoxM1, Cenpf, and Nsd2, which are all aberrantly activated and functionally necessary for aggressive PCa, in both humans and mice (Aytes et al., 2018; Aytes et 
Vasciaveo et al.

al., 2014), as well as activation of the glucocorticoid receptor (Nr3c1), which represents a hallmark of CRPC in human PCa (Arora et al., 2013). Interestingly, subtype $C_{5}$ presented dysregulation of proteins associated with NEPC, including Foxa2 and Foxa1 (Fig. 3A, Fig. S4C) (Baca et al., 2021) and was significant enriched in the Beltran NEPC signature $\left(P<10^{-4}\right.$, see STAR Methods) (Beltran et al., 2016), while no enrichment for the NEPC signature was detectable in subtypes $C_{1}$ $-C_{4}$. Notably, although two of the GEMM-DTs associated with advanced PCA adenocarcinoma and NEPC ( $C_{4}$ and $C_{5}$, respectively) were castrated, all were derived without pharmacological treatments, which is a novel feature of the GEMM cohort compared to a majority of tumors in analogous human PCa cohorts.

To further characterize the GEMM subtypes and to assess their relationship to human PCa, we performed pathway enrichment analyses using $>900$ relevant gene sets, collected by Broad Institute MSigDB (Liberzon et al., 2015), the Kyoto Encyclopedia of Genes and Genomes (KEGG) (Kanehisa and Goto, 2000) and REACTOME (Fabregat et al., 2018) (Table S2D). To determine the top pathways associated with each subtype, we computed the enrichment of each gene set in proteins differentially active in each sample, and then integrated across samples in each cluster using Stouffer's method, see STAR Methods. These analyses revealed that the most aggressive subtypes, $C_{4}$ and $C_{5}$, show strong enrichment in proliferation and oncogenic hallmarks, e.g., G2M Checkpoint $\left(P=2.14 \times 10^{-39}\right)$, E2F Targets $\left(P=4.5 \times 10^{-88}\right)$, DNA Repair $\left(P=5.8 \times 10^{-68}\right)$, and MYC Targets V1 and V2 (integrated $\left.P=3.3 \times 10^{-21}\right)$ (Fig. S4D; Table S2D). (Note that all $p$-values reported in this manuscript are corrected for multiple hypothesis testing, see STAR Methods). Interestingly, interferon and inflammatory response hallmarks, including interferon alpha response $\left(P=2.4 \times 10^{-52}\right)$, interferon gamma response $\left(P=8.2 \times 10^{-68}\right)$ and inflammatory response $\left(p=4.7 \times 10^{-18}\right)$ were downregulated in subtypes $C_{4}$ and $C_{5}$ (Fig. S4D; 
Vasciaveo et al.

Table S2D), suggesting that the most aggressive tumors may have an immunosuppressive tumor microenvironment.

Taken together, these molecular analyses define a series of GEMM subtypes $\left(C_{1}-C_{5}\right)$ that: (i) are distinguished by novel as well as previously known MR protein activities; (ii) model a wide range of PCa phenotypes from indolent to aggressive lethal variants, including castrationsensitive and castration-resistant tumors; and (iii) can differentiate lethal subtypes of adenocarcinoma and NEPC, independent of prior treatment. Thus, this GEMM cohort represents a valuable resource to characterize and model human $\mathrm{PCa}$, particularly for advanced tumors, as would be the principal focus for predicting drug treatments for human patients.

Matching GEMM-DTs to patient tumors and metastases: Having established that the molecular programs of the GEMM cohort are relevant for human PCa, we next asked whether individual GEMM-DTs can provide high-fidelity (cognate) surrogates for individual patients. We thus compared the MR protein activity signature for individual human patients with that of each available GEMM-DT ( $\mathrm{n}=91)$ and designated those presenting highly significant conservation of patient specific MR activity ( $P \leq 10^{-5}$, by 1 -tail aREA test) as cognate models. For these studies, we queried two well-characterized patient cohorts, one comprised of treatment naïve primary tumors collected by The Cancer Genome Atlas (TCGA, $n=333$ ) (Cancer Genome Atlas Research, 2015), and a second comprised of post-treatment metastatic biopsies from mCRPC patients collected as part of the Stand Up to Cancer consortium-Prostate Cancer Foundation CRPC500 cohort (SU2C, $\mathrm{n}=212$ ) (Abida et al., 2019).

First, we assembled distinct human $\mathrm{PCa}$ interactomes for the primary tumors and metastases by ARACNe analysis of RNA-seq profiles from the TCGA and SU2C cohorts, respectively, see STAR methods (Table S3A,B). Then, we used VIPER to transform the transcriptional profiles of the TCGA and SU2C cohorts into protein activity profiles, using their 
Vasciaveo et al.

respective interactomes (Table S3C,D). Differential expression signatures for VIPER analysis were computed using as reference a combination of all RNA seq profiles from the GTEx normal prostate cohort ( $n=245$ ) (Consortium, 2013) and a humanized version of normal prostate tissue from GEMMs (see STAR methods). This approach allowed identification of MR proteins that are specifically dysregulated compared to normal prostate tissue.

To identify cognate models on an individual patient basis, we generated protein activity signatures by comparing each GEMM-DT to the same reference as above. We then assessed the fidelity of each of the GEMM-DTs to each individual TCGA or SU2C patient by assessing the enrichment of the 25 most activated $(25 \uparrow)$ and 25 most inactivated proteins $(25 \downarrow)$ of each human tumor, representing its candidate MR proteins (MR proteins hereafter for simplicity), in proteins differentially active in each GEMM-DT (Fig. 4; Table S4A,B). A fixed number of MR proteins was used for two reasons: first, it is required to make the statistics of enrichment analyses comparable across proteins and cohorts. Second, we have shown that an average of 50 MRs is sufficient to account for the canalization of functionally-relevant genetic alterations in the vast majority of TCGA samples (Paull et al., 2021). For simplicity we refer to these $25 \uparrow+25 \downarrow$ MR proteins as the MR-activity signature. A highly conservative statistical threshold $\left(P \leq 10^{-5}\right)$ was selected to nominate high-fidelity, cognate GEMMs-DTs, see STAR Methods.

Analysis of primary tumors from the TCGA cohort revealed that $78 \%$ had at least one highfidelity cognate GEMM-DT ( $n=261 / 334$; Fig. 4; Table S4A). Strikingly, analysis of PCa metastases from the SU2C cohort revealed an even greater fraction (93\%) of patients with highfidelity cognate models ( $n=198 / 212$; Fig. 4; Table S4B). This likely reflects the inherent bias of the GEMM cohort towards more aggressive $\mathrm{PCa}$ phenotypes and their relative underrepresentation of indolent phenotypes (see Fig. 3). Heatmap representation of the matched patients and GEMM-DTs shows good clustering of patient and GEMM-DTs in the same subtype 
Vasciaveo et al.

$\left(C_{1}-C_{5}\right)$. While most patients were matched to multiple GEMM-DTs, we highlighted the GEMMDTs representing the top 5 most statistically significant MR-based matches of each patient (Fig. 4), since these would provide the most realistic repertoire for preclinical or co-clinical studies. As expected, given the more aggressive nature of SU2C tumors, highest-fidelity models for the TCGA and SU2C cohorts formed distinct clusters. These findings demonstrate that tumors from the GEMM cohort can represent high-fidelity surrogates for most PCa patients, and especially for those with aggressive disease who would most benefit from having cognate models for pre/coclinical validation of novel treatment options in vivo.

Generation of drug perturbational profiles to identify MR-inverter drugs: As discussed above, two PCa cell lines-the androgen dependent LNCaP and the androgen-independent DU145 cell lines - jointly provided high-fidelity models for $>80 \%$ of the tumors in the TCGA cohort, based on MR-activity signature conservation (Douglass et al., 2022). To predict optimal treatments for PCa patients with aggressive tumors, we focused on the DU145 cell line as a model for drug MoA analysis. Specifically, drug MoA was assessed from RNA-seq profiles of DU145 cells harvested at $24 \mathrm{~h}$ following treatment with 117 FDA-approved and 218 late-stage experimental (i.e., in Phase II and III clinical trial) drugs, as well as vehicle control (DMSO) (i.e., $n=335$ drugs in total, Table S5A). To minimize activation of cell death or cellular stress pathways that would confound assessment of drug MoA, cells were treated with the $48 \mathrm{~h} \mathrm{EC}_{20}$ concentration of each drug (i.e., highest sublethal concentration), as assessed from 10-point drug response curves, see STAR Methods. RNA-seq profiles were generated using PLATE-Seq, which was specifically designed to generate profiles of drug perturbations (Bush et al., 2017).

For each drug, differential protein activity profiles, representing the drug's MoA, were then generated by VIPER analysis of drug vs. vehicle control-treated cells, see STAR Methods (Table S5B). Cluster analysis of differentially activated proteins following drug treatment with the most 
bioactive agents-i.e., 115 drugs inducing the most significant differential protein activity patterns -identified 11 drug clusters $\left(D_{1}-D_{12}\right)$ based on differential activation/inactivation of 13 protein sets (programs) $\left(\mathrm{P}_{1}-\mathrm{P}_{13}\right)$ (Fig. S6). Consistent with the analysis, drugs presenting similar MoA were clustered together or in closely related clusters. For instance, cytotoxic drugs cosegregated in clusters $D_{1}, D_{2}, D_{5}, D_{7}$ and $D_{8}$, while kinase inhibitors were found mostly in clusters $D_{3}, D_{10}$, and $D_{12}$, including a majority of MAPK (i.e., sorafenib, dabrafenib, vemurafenib, trametinib), EGFR inhibitors (i.e., lapatinib, erlotinib, vandetanib), and mTor inhibitors (i.e., everolimus, temsirolimus) in $D_{12}$ and $D_{12}$. Similarly, a subset of hormone blockade drugs (i.e., anastrazole, enzalutamide, abiraterone, mitotane) clustered in $D_{6}$ and $D_{9}$, and another subset (i.e., raloxifene, leuprolide, exemestane, tamoxifen) in $D_{3}$ and $D_{4}$. Folate (i.e., methotrexate, pralatrexate) and microtubule inhibitors (i.e., paclitaxel, ixabepilone) clustered in $D_{4}$, while proteasome inhibitors (i.e., ixazomib, carfilzomib, bortezomib) presented very similar profiles in $D_{11}$ and $D_{12}$. In contrast, as expected, more pleiotropic drugs with broad-spectrum MoA, such as HDAC inhibitors (i.e., vorinostat, panobinostat, belinostat), CRBN inhibitors (i.e., thalidomide, lenalidomide, pomalidomide), and de-methylating agents (i.e., decitabine, azacytidine), were broadly distributed across multiple clusters. Given the high reproducibility of replicate perturbational profiles from the same drug $(P \ll 0.05$, by 2 -tail enrichment analysis), for the majority of the 115 bioactive compounds, and the diversity of the differential protein activity they induce, this suggests that the DU145 perturbational profiles effectively inform on drug MoA.

Using Oncoloop to predict candidate drugs for individual patients: To predict candidate drugs, we identified those for which both a patient's and its cognate GEMM-DT's MR-activity signature was significantly inverted in drug-treated vs. vehicle control-treated cells (MR-inverter drugs) (Fig. 5A-C). Since our goal is to identify treatments for patients with advanced, rather than indolent, PCa, we focused on metastatic patients in the SU2C cohort and their cognate GEMM- 
Vasciaveo et al.

DTs (which could be identified for $>90 \%$ of these patients) (see Fig. 4). For each patient and cognate GEMM-DT, MR-inverter drugs were identified by enrichment analysis of the top $25 \uparrow$ and 25 $\downarrow$ MR proteins in the tumor, in protein inactivated and activated in drug-treated vs. vehicle control-treated cells, respectively, at a conservative statistical threshold $\left(P=10^{-5}\right)$ (Table S5C,D). The resulting PGD-loops - comprising a patient, its cognate GEMM-DT, and the candidate MR-inverter drugs (Fig. 5A) - were ranked based on the Stouffer integration of three z-scores, including: (a) $Z_{P G}$, the GEMM vs. patient MR-activity conservation $z$-score (Table S4); (b) $Z_{P D}$, the patient-specific MR-inverter drug z-score (Table S5C); and (c) $z_{G D}$, the cognate GEMM-DTspecific MR-inverter drug z-score (Table S5D). Considering all possible combinations of SU2C patients $(n=212)$, GEMM-DTs $(n=91)$, and drugs $(n=337)$, there were $>6.5$ M potential PGDloops; of these 668,138 achieved statistically significant p-values $\left(P \leq 10^{-5}\right)$ on all three $z$-scores (Table S6). On average, 48 cognate GEMM-DTs were identified as significant cognate models $\left(P \leq 10^{-5}\right)$ for each SU2C tumor, and 22 FDA-approved drugs were identified as candidate MRinverters $\left(P \leq 10^{-5}\right)$ for each SU2C tumor/cognate-GEMM-DT pair. Such extensive coverage supports the use of OncoLoop in a co-clinical setting.

To provide a visually-interpretable representation, we show three heatmaps reporting the statistical significance of model fidelity and drug MR-inversion analyses for a subset of SU2C patients $(n=56)$, GEMM-DTs $(n=5)$, and drugs $(n=28)$, which were especially enriched in statistically significant PGD-Loops (Fig. 5A). For illustrative purposes, we highlight a PGD-loop defined by an mCRPC patient (SC_9182_T), its cognate GEMM-DT (CMZ315)-harboring mutation of $\mathrm{p53}$, a gene that is frequently dysregulated in human CRPC (see Fig. S1) - and the drug trametinib (Fig. 5B). Notably, all three Normalized Enrichment Score (NES) values for this PGD-Loop were highly significant, including for the model fidelity analysis (NES $=8.14, P=$ $4.1 \times 10^{-16}$ ), as well as for the patient-specific (NES $=-5.47, P=4.4 \times 10^{-8}$ ), and cognate GEMM- 
Vasciaveo et al.

DT-specific (NES $=-4.99, P=6.2 \times 10^{-7}$ ) trametinib-mediated MR-inversion (Fig. 5C), resulting in a highly significant integrated $p$-value (NES $\left.=10.71, P=4.6 \times 10^{-27}\right)$.

Preclinical validation: To optimize the translation of this approach to clinical practice, we focused on PGD-Loops comprising drugs that were both clinically-available and most recurrently predicted for SU2C patients (Fig. 6A). Specifically, we considered only FDA approval drugs ( $n=117 / 335)$, predicted for $\geq 50 \%$ of the SU2C tumors $(n=37 / 117)$, and tested at a physiologically-relevant concentration $(\leq 1 \mu \mathrm{M})$, ultimately resulting in 16 candidate drugs for follow-up validation. Cluster analysis of their MR-inversion z-score for individual SU2C tumors (i.e., predicted drug sensitivity) identified 5 clusters, which represent subsets of patients predicted to be sensitive to the same drugs (i.e., pharmacotypes; See STAR Methods) (Fig. 6A). Concurrent, yet fully independent stratification of the GEMM-DTs, using the same approach, while not limiting coverage to $\geq 50 \%$, identified the same top 16 drugs, thus confirming the representative nature of these models. (Fig. 6B).

Final selection for in vivo validation eliminated drugs with overlapping MoA (e.g., multiple HDAC inhibitors) or lacking demonstrated variable efficacy in prior PCa clinical trials, thus yielding four candidates: temsirolimus (m-TOR inhibitor) (NCT00919035, NCT00012142) (Kruczek et al., 2013); trametinib (MEK inhibitor) (NCT02881242, NCT01990196), panobinostat (HDAC inhibitor) (NCT00667862, NCT00878436) (Rathkopf et al., 2013), and bortezomib (proteosome inhibitor) (NCT00193232, NCT00183937). As a negative control, we selected cabazitaxel-the current standard of care for advanced $\mathrm{PCa}$-which was predicted as a significant MR-inverter for the human tumors but not for the cognate GEMM-DTs (see Fig. 6B).

To test drug sensitivity predictions, we performed tumor growth assays in allografts derived from two cognate GEMM-DTs, namely CMZ315 and CMZ150 represented in a majority of the significant PGD-Loops and representing two of the aggressive GEMM clusters, $C_{3}$ and $C_{4}$, 
Vasciaveo et al.

respectively. These were derived from a p53-mutated and a MYC-amplified mCRPC GEMMs, respectively (Fig. 6C-F; Fig. SA-D). For each drug, we used the published conditions for in vivo mouse studies to determine the appropriate concentration and treatment schedule, and we also confirmed their uptake into allograft tumors, by pharmacokinetic assays, see STAR Methods (Fig. S7A). Three of the four predicted drugs - namely temsirolimus, trametinib, and panobinostatinduced significant tumor volume and weight reduction in allografts from both cognate GEMMDTs ( $P \leq 0.01$, one-way ANOVA, Fig. 6C-F; Fig. S7B-D). Bortezomib did not significantly inhibit tumor growth in either allograft, potentially due to cell adaptation mechanisms that buffered the initial MR-inversion. Also consistent with predictions, cabazitaxel induced only modest tumor volume/weight reduction, which was significant in only one of the two allografts. Taken together, these findings confirm that drug-mediated MR-inversion is frequently (75\% of treated allografts) associated with loss of tumor viability in vivo.

Lastly, we performed pharmacodynamic studies to assess their ability to recapitulate the predicted MR-inversion (Fig. 6G). Specifically, to assess MR-activity inversion before significant tumor cell death or necrosis could ensue, we analyzed RNA-seq profiles from short-term (5-day) drug- vs. vehicle control-treated allograft (CMZ315). Confirming DU145-based predictions, all four predicted drugs induced highly significant MR-inversion in vivo, as evidenced by GSEA analyses showing significantly negative NES values $\left(P \leq 10^{-7}\right.$, for all tested drugs, by 1 -tail aREA test) (Fig. $6 G)$.

Co-clinical validation in a human-relevant context: To assess whether OncoLoop predictions may be further translated to human tumor contexts, we performed analogous studies using the well-characterized LuCaP series of PDX models, which were established from primary PCa and PCa metastases obtained from University of Washington Tissue Acquisition Necropsy program (Nguyen et al., 2017). Notably, the LuCaP PDX models were developed from biologically 
heterogeneous advanced PCa tissues from primary and metastatic sites and include a range of tumors that model CRPC, and vary in their response to castration, with some models displaying castration-sensitivity (Nguyen et al., 2017).

To assess their relevance in a co-clinical study context, we generated RNA-seq profiles of 6 LuCaP PDX models perturbed with multiple drugs (see STAR Methods) and used them to generate a PDX interactome (Table S7A). Among all 6 baseline LuCaP PDX models, VIPER analysis identified LuCaP-73 as the most significantly matched to the GEMM-DTs used in the preclinical studies (Fig. 7A). Furthermore, among the candidate drugs identified for the human tumors (see Fig. 6A), several were identified as significant MR-inverters for the LuCaP-73 PDX, including trametinib and panobinostat (Fig. 7B; Table 7B). Therefore, we tested whether these two drugs could abrogate LuCaP-73 viability in vivo. Indeed, both drugs showed near-complete tumor growth inhibition $(P<0.0001$; Fig. $7 C-E)$. These findings provide important support for the translation of OncoLoop to a human PCa context. 
Vasciaveo et al.

\section{Discussion}

Predicting targeted and chemotoxic drug activity in human patients remains a key challenge in precision medicine and approaches to identify preclinical models that best recapitulate patient-relevant drug response have also been elusive. To address these challenges, we have developed quantitative protein activity-based metrics to identify both high-fidelity cognate models-predicted to recapitulate patient-specific drug sensitivity-as well as to predict and validate drug sensitivity for both the human and its cognate model's tumor. The resulting OncoLoop framework allows rapid evaluation of drugs predicted to be optimal inverters of MR activity in a human tumor using quantitatively-defined cognate models to support rapid-turnaround co-clinical studies. Notably, this approach can also be applied to evaluate candidate drugs for clinical trials, including umbrella and basket trials, based on in vivo validated drug stratification across models representing distinct pharmacotypes (Fig. 7F). Thus, OncoLoop represents an accurate and versatile framework for both predicting and evaluating individualized drug treatments in real-time.

We demonstrate the utility of OncoLoop in the context of PCa, which has many features that make it amenable to this approach. Leveraging an extensive and diverse collection of GEMMs, we find that $>90 \%$ mCRPC patients in a published PCa cohort are represented by at least one cognate GEMM-DTs. Further, drugs predicted to revert the MR activity of both the patient and its cognate model were experimentally validated both in allografts from the GEMMDT as well as in a cognate PDX model, and they also recapitulated their predicted MR-inversion potential in vivo. These findings demonstrate that OncoLoop can be used to predict and evaluate drug treatments for patients. Notably, all of the resources described herein are available, and therefore can be applied in clinical practice. 
Vasciaveo et al.

Nonetheless, despite its potential value, there are also several caveats that will benefit from further refinements. First, focusing only on clinically relevant drugs, either FDA-approved or in Phase II/II trials, may not be ideally suited to the approach, especially as single agents, since almost none of these drugs were designed to target MR proteins. In future studies, extension to additional experimental agents may expand the repertoire of effective drugs, especially considering the new classes of PROTACS (Bekes et al., 2022) and antisense agents (Crooke et al., 2018). Additionally, drug perturbation profiles are limited by availability of appropriate cell lines MR-matched to human patients. For example, the current study does not adequately inform on drugs that target NEPC tumors since neither the LNCaP nor the DU145 cell line recapitulate the MR-activity signature of this subtype. This can be addressed by generating drug perturbation profiles from primary, NEPC patient derived cells or organoids. Moreover, despite the encouraging results reported here, MR signature analysis and MR-inverter drug predictions are not $100 \%$ accurate, as is the case for most machine learning methods. We thus envisage that larger scale validation will be required before OncoLoop predictions may be confidently used in the design of co-clinical, basket, or umbrella clinical trials.

Finally, a key caveat is the largely heterogeneous nature of most tumors, which present molecularly distinct subpopulations with potentially equally distinct drug sensitivity. Thus, when used to analyze bulk tumor profiles, OncoLoop may miss nominating drugs targeting the less represented subpopulations, thus selecting for drug-resistant ones and ultimately leading to relapse. A possible way to overcome this is to perform OncoLoop analyses at the single cell level, which should enable prioritization of drugs for all detectable subpopulations (Ding et al., 2019). A second one is to perform analysis on the post-treatment minimal residual tumor mass, which is likely highly enriched for resistant subpopulations (Echeverria et al., 2019; Risom et al., 2018). Additionally, while OncoLoop predictions are transcriptome based, recent results show that 
Vasciaveo et al.

additional omics modalities, such as a patient's mutational profile and protein structure, among others, can be readily integrated to further refine MR protein identification and drug prediction (Paull et al., 2021).

Beyond PCa, OncoLoop is readily generalizable to predicting both cognate models and drug sensitivity for other cancer types, as well as in non-cancer-related contexts. Notably, largescale human, GEMM, and/or PDX cohorts are available for most cancer types. In addition, we have already generated genome-wide perturbational profiles in a constantly growing number of cell lines, already comprising representatives for 23 distinct tumor subtypes (see (Douglass et al., 2022) for partial coverage of the PanACEA database). Critically, the ability to stratify drug sensitivity in precisely identified molecular subtypes (pharmacotypes), if further validated, may lead to rapid design of basket and umbrella trials, including using adaptive design approaches to efficiently replace baskets that fail to validate.

Outside of cancer, MR-based predictions have been validated in disease as different as Parkinson's (Brichta et al., 2015), ALS (Mishra et al., 2020), alcohol dependency (RepunteCanonigo et al., 2015), and Type II diabetes (Son et al., 2021), suggesting direct OncoLoop applicability to such contexts, as long as appropriate drug-perturbation data were available. Indeed, while human translation of drugs validated in a GEMM or PDX context has been reasonably effective in cancer, mouse models have largely failed to recapitulate the effect of drugs on human disease. In this context, OncoLoop's quantitative fidelity metrics may help identify more appropriate mouse models for drug validation.

The current design of OncoLoop relies on MR-based predictions of model similarity and drug sensitivity. However, alternative approaches could also be tested using the same framework presented here. For instance, transcriptome-based approaches have shown promising results in predicting sensitivity of human patients in a clinical context (Rodon et al., 2019), while neural 
network-based methods trained on multi-omics data have shown promising results in translating drug sensitivity assays from a training set of cell lines and mouse models to an independent test set of PDX models (Ma et al., 2021). Similarly, transcriptome-based approaches for assessing model fidelity have also been proposed (Warren et al., 2021).

Taken together, our data shows that OncoLoop may provide a valuable contribution to the emergent field of precision medicine, by complementing rather than supplanting existing approaches based on its ability to couple effective drug and high-fidelity model predictions. 
Vasciaveo et al.

\section{STAR Methods}

\section{A resource of genetically engineered mouse models (GEMM) of prostate cancer}

All experiments using animals were performed according to protocols approved by the Institutional Animal Care and Use Committee (IACUC) at Columbia University Irving Medical Center (CUIMC). As described in Table S1A, the genetically engineered mouse models (GEMMs) used in this study utilize the $N k x 3.1^{\text {CreERT2l+ }}$ allele to activate an inducible Cre recombinase in the prostatic epithelium (Wang et al., 2009). The Nkx3.1CreERT2/+ allele was crossed with various other mouse alleles to achieve conditional deletion or conditional activation in the prostatic epithelium, or with the Hi-Myc transgene in which MYC expression is regulated by a constitutively active Probasin promoter (Ellwood-Yen et al., 2003). For lineage tracing to visualize tumors and metastases ex vivo, mice were further crossed with a conditionally-activatable fluorescent reporter allele (Rosa-CAG-LSL-EYFP-WPRE) (Madisen et al., 2010). All mice were maintained on a predominantly C57BL/6 background; since the focus of our study is prostate cancer, only male mice were used. Several of these GEMMs have been described previously (Arriaga et al., 2020; Aytes et al., 2013; Floc'h et al., 2012; Wang et al., 2012; Zou et al., 2017). All multi-allelic strains are available from the Jackson laboratory (Table S1A).

Phenotypic analysis: All studies were performed using littermates that were genotyped prior to tumor induction. Mice were induced to form tumors at 2-3 months of age by administration of tamoxifen (Sigma-Aldrich, St. Louis, MO) using $100 \mathrm{mg} / \mathrm{kg}$ (in corn oil) once daily for 4 consecutive days. Following tamoxifen-induction, mice were monitored three times weekly, and euthanized when their body condition score was $<1.5$, or when they experienced body weight loss $\geq 20 \%$ or signs of distress, such as difficulty breathing or bladder obstruction. Control (non-tumor 
Vasciaveo et al.

induced) mice received only vehicle (i.e., corn oil) and were otherwise monitored in parallel with tumor-induced mice. As indicated, surgical castration was performed at 1-10 months after tumor induction (Table S1A). Where indicated, tumor volume was monitored by Magnetic Resonance Imaging (MRI) using a Bruker Biospec 9.4T Tesla Small Animal MR Imager located within the mouse barrier facility in conjunction with the Oncology Precision Therapeutics and Imaging Core (OPTIC) of the Herbert Irving Comprehensive Cancer Center (HICCC). Images were generated using ParaVision 6 software for preclinical MRI (Bruker) and reviewed by an MRI physicist. Volumetric analysis was done using 3DSlicer software (http://www.slicer.org).

At the time of sacrifice, tissues were collected and YFP-positive prostatic tumors and metastases were visualized by ex vivo fluorescence using an Olympus SZX16 microscope (Ex490-500/Em510-560 filter). For histopathological analysis, tissues were fixed in 10\% formalin (Fisher Scientific, Fair Lawn, NJ), and hematoxylin and eosin (H\&E) and immunostaining were done using $3 \mu \mathrm{m}$ paraffin sections as described (Arriaga et al., 2020; Zou et al., 2017). Antibodies used in these studies were: Androgen receptor (Abcam ab133273, 1:200; Cambridge, MA), Ki67 (eBioScience 14569882, 1:500; San Diego, CA), Pan-cytokeratin (Dako Z0622; 1:500; Santa Clara, CA) and Vimentin (Cell Signaling 5741S, 1:200; Danvers, MA). Images were captured using an Olympus VS120 whole-slide scanning microscope. Histopathological scoring of GEMM prostate cancer phenotypes was assessed independently by two pathologists (AR and $\mathrm{SdB}$ ) based on evaluation of both the H\&E-stained and adjacent immuno-stained tissues; these analyses are provided in Table S1A,B.

Generation of allografts: Following protocols approved by IACUC at CUIMC, allografts were generated by transplanting freshly dissected prostate tissues from the GEMMs subcutaneously into the flank of male Athymic nude mice (Hsd:Athymic Nude-Foxn1nu, Envigo, Boyertown PA). Briefly, at the time of euthanasia, tumors were collected and cut into $<2 \mathrm{~mm}$ 
Vasciaveo et al.

pieces. Tissues were rinsed twice in cold sterile Dulbecco's Modified Eagle Medium (DMEM; Thermo Fisher Scientific, Waltham, MA), soaked in Matrigel (Corning; Corning, NY), and implanted into the flanks of male hosts. Allografted tumors were harvested when the tumor size reached $2 \mathrm{~cm}$ or earlier if the body condition score of the host mouse was $<1.5$ or the mice exhibited signs of distress. At time of harvesting, allografted tumors were passaged to new hosts and/or snap frozen in $80 \%$ DMEM, $10 \%$ fetal bovine serum (FBS, Gemini Bio-Products, West Sacramento, CA, USA), and 10\% DMSO. Alternatively, the harvested allografts were fixed in 10\% formalin for histopathological analyses, as above. A summary of allografted tumors is provided in Table S1A; as part of this GEMM resource, allografted tumors will be made available upon request by contacting the authors.

Generation of organoids: Mouse tumor organoids were generated as described (Chua et al., 2014). Briefly, freshly dissected prostate tissues were dissociated in DMEM containing 5\% FBS and $1 x$ collagenase/hyaluronidase (STEMCELL Technologies, Vancouver, BC, Canada), followed by trypsinization with $0.25 \%$ trypsin-EDTA (Gibco, Carlsbad, CA) at $4{ }^{\circ} \mathrm{C}$ for $1 \mathrm{~h}$. Trypsinization was stopped by addition of Hanks' Balanced Salt Solution (HBSS) media (STEMCELL Technologies) containing $2 \%$ FBS and the tissue was further dissociated by treatment with dispase $(5 \mathrm{mg} / \mathrm{mL}) / D N a s e l(0.1 \mathrm{mg} / \mathrm{mL})$ solution (STEMCELL Technologies). The cell suspension was filtered using a $40 \mu \mathrm{m}$ cell strainer (Falcon; Corning, NY, USA), resuspended in HBSS containing $2 \%$ FBS (Gemini Bio-Products, West Sacramento, CA) and subjected to fluorescence activated cell sorting (FACS) using a BD-FACS Aria cell sorter (BD Biosciences, Franklin Lakes, NJ) to collect the YFP-lineage marked prostate cells. Sorted cells were plated in low-attachment 96 -well plates at 10,000 cells per well and cultured in organoid culture medium containing: hepatocyte medium (Corning) with 1x Glutamax (Gibco), 10 ng/mL EGF (Corning), 10 $\mu \mathrm{M}$ ROCK inhibitor (STEMCELL Technologies), 5\% heat-inactivated charcoal-stripped FBS 
(Thermo Fisher Scientific), $100 \mathrm{nM}$ DHT (Sigma-Aldrich), 5\% Matrigel (Corning) and 1x antibioticantimycotic (Gibco). Organoids were harvested by centrifugation and embedded with Histogel (Thermo Fisher Scientific) (Chua et al., 2014), and processed for histopathological analyses, as above. A summary of organoids is provided in Table S1A. Although in the current study preclinical validation focused was done using the allograft models in vivo, as part of the GEMM resource, the mouse organoids will be made available upon request by contacting the authors.

\section{Transcriptomic analysis of GEMMs}

RNA-seq data was generated from $n=136$ GEMM-derived tumors (GEMM-DTs) and GEMM-derived normal prostate tissue (GEMM-DP). RNA was prepared from snap-frozen samples by homogenization in TRIzol Reagent (Invitrogen; Waltham, MA) using a Fisherbrand ${ }^{\mathrm{TM}}$ 150 Homogenizer (Fisher Scientific, Pittsburg, PA Catalog number 15-340-168) followed by extraction using the MagMAX-96 total RNA isolation kit (ThermoFisher Scientific, Waltham, MA, USA). Total RNA was enriched for mRNA using poly-A pull-down; only RNA samples having between $>200 \mathrm{ng}$ and $1 \mu \mathrm{g}$ and with an RNA integrity number $(\mathrm{RIN})>8$ were used. Libraries were made using an Illumina TruSeq RNA prep-kit v2 or TruSeq Stranded mRNA library prep kit, and sequenced using an Illumina HiSeq2500/4000 or NovaSeq6000. RNA-seq profiles were generated by mapping mRNA reads to the mouse reference genome (version GRCm38 mm10), using kallisto v0.44.0 (Bray et al., 2016). Sequence-based bias correction was applied to pseudoalign the reads and quantify transcripts abundance in each sample. The resulting raw reads were finally mapped using the ENSEMBL gene model and summarized to transcript per million (TPM) to remove gene length bias thus allowing for inter and intra-sample comparison between genes. Raw and processed data were deposited in GEO (GSE186566). 
Network analysis: A GEMM PCa-specific regulatory network (interactome) was reverse engineered from the resulting GEMM-DT and GEMM-DP RNA-seq profiles $(n=136)$ using ARACNe-AP (Lachmann et al., 2016), the most recent implementation of the ARACNe algorithm (Basso et al., 2005) (Table S2A). Default values for the algorithm were used for the number of bootstraps $(n=200)$, Mutual Information (MI) $P$-value threshold $\left(P \leq 10^{-8}\right)$, and Data Processing Inequality (DPI) (enabled $=$ yes). A total of $n=3,163$ regulatory proteins were selected from Gene Ontology (GO)-generated protein sets, including $n=1,046$ Transcription Factors (TF) - "DNAbinding transcription factor activity" (GO:0003700) - and $n=2,217$ co-Transcription Factors and chromatin remodeling enzymes-"Transcription coregulator activity" (GO:0003712), "DNA binding” (GO:0003677), "Transcription factor binding” (GO:0008134), "Regulation of transcription, DNA-templated" (GO:0006355), "Histone binding" (GO:0042393), and "Chromatin organization" (GO:0000790) -see (Ashburner et al., 2000; Margolin et al., 2006).

GEMM-DT analyses: For all subsequent analyses, we selected a subset of $n=91$ GEMMDTs that most faithfully recapitulated pathophysiologically-relevant prostate cancer phenotypes, based on their histopathology (Table S1B).

Interactome comparison: The expression matrix of the GEMMS was converted to gene expression signatures using Median Absolute Deviation (MAD) and independently analyzed with VIPER using both a prior GEMM-specific interactome (Aytes et al., 2014), generated from Illumina gene expression microarrays data, $I_{\text {lold, }}$ and the new interactome described above, $I_{\text {New. }}$ For each GEMM-DT sample $(n=91)$ the 25 most activated $(25 \uparrow)$ and 25 most inactivated $(25 \downarrow)$ MRs were identified-using a differential expressed signature obtained by comparing each sample to the dataset's centroid. The mean of the absolute Normalized Enrichment Score (NES) across all 50 MRs was used to assess the interactome's ability to identify sample-specific, statistically significantly differentially active proteins. The NES probability densities and expected values were 
Vasciaveo et al.

also computed and plotted (Fig. S4A), showing that the new interactome is more effective in identifying differentially active proteins compared to the prior one $\left(P<2.2 \times 10^{-16}\right.$, as assessed by a two-sample Kolmogorov-Smirnov test).

GEMM-DT subtype identification: We used the VIPER algorithm (Alvarez et al., 2016) to transform the RNA-seq profile of each GEMM-DT into a differential protein activity profiles, using regulatory protein targets (regulons) captured by the GEMM interactome. To avoid bias due to different regulon sizes, regulons were pruned to include only the 50 highest likelihood targets, as recommended in (Alvarez et al., 2016), and regulons with $<50$ targets were excluded from the analysis. From 2,858 regulatory protein regulons recovered by ARACNe inference, only 64 were removed due to inadequate regulon size. The resulting regulatory network comprising regulons of 2,794 regulatory proteins was used for subsequent GEMM-related analysis.

Since this analysis was aimed at stratifying disease subtypes by identifying the proteins that are most differentially active between subtypes, we generated sample-specific gene expression signatures (GES) based on the z-score of differentially expressed genes in each GEMM-DT vs. the average of all GEMM-DTs as the reference distribution. This approach is designed to maximize intra-cohort differences. Results are summarized in Table S2B.

We performed Uniform Manifold Approximation and Projection (UMAP) (Mclnnes et al., 2018) for dimensionality reduction analysis on the 91 GEMM-DT protein activity profiles, after selecting the most statistically significant principal components (PCs), by applying the JackStraw algorithm (Chung and Storey, 2015). Six statistically significant PCs were identified $(P \leq 0.01)$ and used for downstream analysis. Next, we built a Shared Nearest Neighbor (SNN) Graph (Houle et al., 2010) using the Seurat v3.1.2 (Stuart et al., 2019) implementation of the algorithm. For the SSN algorithm we used $k=7$ as number of neighbors for graph construction. Finally, cluster analysis was performed using the Louvain algorithm (Blondel et al., 2008). To select the optimal 
resolution parameter for the Louvain algorithm, we ran a grid search across the range $[0.01,2]$ in steps of 0.05 and used the average Silhouette Score (Rousseeuw, 1987) and co-segregation of clinical parameters (e.g., metastatic samples, NEPC samples, etc.) across the possible cluster solutions as cost function to optimize the number of clusters. The resulting data are provided in Table S2C. The optimal 5-cluster solution was found at a resolution $\rho=0.41$. Each cluster should thus represent a molecularly distinct GEMM-DT subtype.

To identify the proteins that were most differentially active in each subtype-i.e., representing candidate, subtype-specific Master Regulator (MR) proteins - we integrated the zscores representing the VIPER-measured differential activity of each protein across all subtype samples having silhouette score $>0.25$, using Stouffer's method. To avoid clutter, we visualized the 10 most activated proteins for each subtype $(P \leq 0.01$, Bonferroni Corrected $(B C)$ ) as representative candidate positive MRs (Fig 3A and Table S2C).

Pathway analysis: Gene sets representing canonical pathways and Cancer Hallmarks were selected from the Broad MSigDB (Liberzon et al., 2015), KEGG (Kanehisa and Goto, 2000), and REACTOME databases (Fabregat et al., 2018), see http://www.gseamsigdb.org/gsea/msigdb/collections.jsp. To convert murine genes to human genes, for pathway analysis, gene identifiers were mapped to their human-mouse orthologs using the $\mathrm{R}$ biomaRt services (Smedley et al., 2015). Gene Set Enrichment Analysis (GSEA) was then computed using the analytical Enrichment Analysis Algorithm (aREA) function from the R VIPER package (Alvarez et al., 2016) and used to assess the enrichment of each pathway or Cancer Hallmark gene set in genes encoding for proteins that were differentially active in each cluster. Gene sets with $<20$ or $>250$ genes were excluded as statistically underpowered or unspecific, respectively. Enrichment results are shown in Table S2D. 
Vasciaveo et al.

\section{Patient-Derived xenograft (PDX) model analysis}

Following protocols approved by IACUC at CUIMC, LuCaP PDX lines 73, 77, 78, 81 and 147 (Nguyen et al., 2017) were continuously maintained by passage in male CIEA NOG mice (Taconic, Germantown, New York, USA). Xenografts were harvested when the tumor size reached $2 \mathrm{~cm}$ or earlier if the body condition score of the host mice were $<1.5$ or if they exhibited signs of distress. When the Xenograft tumors were $7-8 \mathrm{~mm}$ in diameter, the host mice were castrated or left intact (mock surgery). To generate additional variability for effective interactome assembly, three days later, the mice were treated with either vehicle or one of 13 selected perturbagens described in (Aytes et al., 2014), once daily, for 5 days. On the afternoon of the fifth day of treatment, mice were euthanized and tumors were collected and snap-frozen in liquid nitrogen, for a total of 140 samples (5 models, 14 treatments, 2 castration states).

RNA extraction and library preparation were performed as described for the GEMM-DT cohort and libraries were sequenced on the Illumina HiSeq2000 instrument. We used RTA (Illumina) for base calling and CASAVA (version 1.8.2) for converting BCL to fastq format, coupled with adaptor trimming. Reads were mapped to a reference genome (Human: NCBI/build37.2) using Tophat (version 2.0.4) (Trapnell et al., 2009) with 4 mismatches (--read-mismatches $=4$ ) and 10 maximum multiple hits $(--$ max-multihits $=10)$. The relative abundance (aka expression level) of each gene and splice isoform was estimated using cufflinks (version 2.0.4) (Trapnell et al., 2010) with default settings. Expression profiles for both the raw and processed data have been deposited in GEO (GSE184427). A Xenograft interactome was generated from the 128 highest quality xenograft-derived gene expression profiles using ARACNe-AP (Lachmann et al., 2016), as described for the GEMM cohort. Results are provided in Table S7A. 
Vasciaveo et al.

\section{Human patient cohort analysis}

For the analysis of human tissue from PCa tumors and normal prostate, we collected $n=790$ RNA-seq profiles from published sources, including profiles from: (a) 245 normal prostate tissues from the GTEx consortium (version 8, date 2017-06-05) (Consortium, 2013); (b) 333 treatment-naïve, clinically-annotated primary prostate adenocarcinoma samples in The Cancer Genome Atlas (TCGA) PCa cohort (Cancer Genome Atlas Research, 2015); and (c) 212 metastatic biopsies from heavily pretreated mCRPC patients from the Stand Up to Cancer cohort (SU2C) (Abida et al., 2019). In addition, we refer to a cohort of 49 metastatic biopsies from patients with castration-resistant adenocarcinoma (adeno-CRPC) or neuroendocrine prostate cancer (NEPC) from which a NEPC signature was derived as discussed in (Beltran et al., 2016).

TCGA interactome: To generate the primary prostate tumor interactome we used data from the full set of 498 patients in the TCGA PCa cohort (Cancer Genome Atlas Research, 2015). RNA-Seq counts data were downloaded from https://portal.gdc.cancer.gov/repository using TCGAbiolinks for R (Colaprico et al., 2016). Regulatory network analysis was performed as described for the GEMM interactome, using a total of $n=2,554$ regulatory proteins were selected into manually curated protein sets, including $n=1877$ Transcription Factors (TF) (GO:0003700) and $n=677$ co-Transcription Factors or chromatin remodeling enzymes (GO:0003712), see (Ashburner et al., 2000; Margolin et al., 2006) Data are provided in Table S3A. The interactome was then generated as described for the GEMM-DT samples.

SU2C interactome: RNA-seq profiles of this cohort were collected as FPKM measurements, as released in the 2019 SU2C-mCRPC dataset (Abida et al., 2019). We restricted our analysis to $n=212$ patients for which RNA-seq data had been generated using the Agilent SureSelect Human All Exon V4 reagents. FPKM were transformed to TPM and regulatory network generation was performed as described above. Data are provided in Table S3B. 
Generation of differential gene expression signatures: To identify gene expression signatures of tumorigenesis for protein activity analysis -including for MR-based patient/GEMMDT matching and to identify MR-inverter drugs-tumor samples from each cohort ( $n=333$ for TCGA and $n=212$ for SU2C), was compared to the same reference control dataset, comprising RNA-seq profiles of 245 normal human prostates from the GTEx cohort, as above, and 26 normal murine prostates, following mouse-to-human gene conversion. The latter were obtained from wildtype and Nkx3.1 heterozygous and homozygous null mice (Table S1B). To allow sequencing depth-independent comparison of tumor vs. control samples, including for low-depth PLATE-seq profiles ( 1M reads), we normalized each sample to an equivalent library size of $10^{6}$ reads, after filtering the data to retain only genes represented across all analyzed datasets, followed by Log transformation. We used the R limma package (Ritchie et al., 2015) to fit a linear model to each gene using the control dataset as reference. Next, for each gene, we computed $P$-values and moderated t-statistics using the empirical Bayes method with parameter robust set to TRUE. For each sample, we used a vector of these statistics to generate a $z$-score vector representing the differential expression of each gene in a tumor sample versus reference control (signature). The GEMMs cohort was processed identically, after cross-species conversion of gene identifiers, which was done as described in the Pathway analysis section above.

Generation of protein activity profiles: Differential protein activity was measured by VIPER analysis of differential gene expression signatures-i.e., each tumor vs. the average of the normal samples control reference-using the corresponding cohort-specific regulatory network. All cohorts were processed using the same parameters. Protein activity profiles are provided in Tables S3C,D. 
Vasciaveo et al.

\section{OncoMatch analysis}

To assess the fidelity of an individual GEMM, cell line or PDX (i.e., tumor models) to an individual human tumor sample, we used their protein activity signatures to compute the NES of the $25 \uparrow+25 \downarrow$ most differentially active proteins (MR-signature, hereafter), as assessed by VIPER analysis of a human sample, in proteins differentially active in the model sample, using the aREA algorithm, as shown in (Alvarez et al., 2019). A fixed MR number was selected to avoid bias due to set size in assessing the NES, while the specific number (i.e., $\mathrm{n}=50$ ) was selected based on the results of a recent pancancer study (Paull et al., 2021) where an average of 50 MR was found to be sufficient to canalize the effect of the functionally relevant genetic alterations, on a sampleby-sample basis, across 20 TCGA cohorts. Murine proteins were humanized (i.e., mapped to human proteins), as described in the Pathway analysis section, via custom $\mathrm{R}$ functions to query the BiomaRt servers (Smedley et al., 2015). The aREA algorithm (Alvarez et al., 2016) was then used to estimate Normalized Enrichment Scores (NES) that were finally converted to $P$-values. The conservative Bonferroni method was used to correct for multiple hypothesis testing and the value $S_{F}=-\log _{10} P$ was used as an MR conservation-based fidelity score. Tumor models with fidelity score $S_{F} \geq 5$ (i.e., $P \leq 10^{-5}$ ) were then selected as high-fidelity cognate models.

For drug validation purposes, the GEMM-DTs with the highest, statistically significant fidelity score $\left(P \leq 10^{-5}\right)$ were used as optimal models for patient-relevant drug validation. All available GEMM-DTs ( $n=91$ ) were tested as potential high-fidelity models of all TCGA and SU2C cohort samples. Results are provided in Tables S4A and S4B, respectively, and in Figure 4, where we show both the top 5 highest fidelity models per patient, as well as all additional statistically significant models $\left(P \leq 10^{-5}\right)$. 
Vasciaveo et al.

\section{Human PCa cancer cell line selection}

To generate drug perturbational profiles, we first identified cell lines representing the highest fidelity models of TCGA PCa samples. Cell Line RNA-seq profiles were downloaded from the Broad Institute web portal (https://data.broadinstitute.org/ccle/) from the Cancer Cell Line Encyclopedia (CCLE) as transcript per million (TPM) measurements with timestamp version 20180929. From these data, which comprise the RNA-seq profiles of 1,019 cell lines, we generated gene expression signature (GES) representing the z-score of genes differentially expressed in each of 8 PCa cell lines in CCLE against the average of all CCLE cell lines as a control reference. Differential gene expression signatures were then VIPER transformed into differential protein activity profiles using the metaVIPER algorithm (Ding et al., 2018) to select either the TCGA or the SU2C interactome as the optimal human PCa network on a protein-byprotein basis.

For androgen-dependent tumors, LNCaP emerged as an optimal model $\left(P \leq 10^{-5}\right)$ for $>70 \%$ of the indolent TCGA samples. The DU145 cell line, while matching a much smaller fraction of the TCGA samples was identified as an optimal match $\left(P \leq 10^{-5}\right)$ for TCGA samples presenting high Gleason Score, low AR activity, and high CENPF and FOXM1 activity-two proteins previously reported as synergistic determinants of malignant PCa (Aytes et al., 2014), which were also aberrantly activated in this cell line. These cell lines were thus selected for drug perturbation assays.

Dose-response curve (DRC) generation: To determine the maximum sublethal concentration of each drug-defined as its $48 \mathrm{~h} \mathrm{EC}_{20}$ concentration-cells were seeded onto 384well tissue culture plates (Greiner 781080 Monroe, NC; columns 1-24, $50 \mu \mathrm{L}$ volume) at a density of 1,000 cells per well. Plates were incubated at room temperature for 30 minutes, and then overnight in an incubator $\left(37^{\circ} \mathrm{C}, 5 \% \mathrm{CO}_{2}\right)$. The next morning (approximately 12 hours after 
Vasciaveo et al.

seeding) compounds were added using the Echo 550 acoustic dispenser. After $40 \mu \mathrm{L}$ of media were removed from each well, each drug was dispensed in ten doses, with each plate run in triplicate. After 48hrs, $25 \mu \mathrm{L}$ of CellTiter-Glo (Promega Corp, Madison, WI) were added to each well. Plates were shaken for 5 minutes before enhanced luminescence readout (EnVision, Perkin Elmer, Shelton, CT). All values were normalized by internal controls contained on each plate and analyzed for $\mathrm{EC}_{20}$ determination using Pipeline Pilot, Dassault Systems.

Drug perturbation analyses: Compared to DRC generation, more cells were needed to isolate sufficient total RNA-seq profile generation. For this purpose, cells were thus seeded onto 384-well tissue culture plates, as above, at a density of 4,000 cells per well. The next morning (approximately $12 \mathrm{~h}$ later), compounds were added using the Echo 550 system as above. Each drug was dispensed at the smaller of its previously determined $\mathrm{EC}_{20}$ or its calculated $\mathrm{C}_{\max }$ (maximum tolerated serum concentration), but never above $10 \mu \mathrm{M}$. The $\mathrm{C}_{\max }$ was used to avoid perturbing cells with non-physiologically relevant drug concentrations. After 24 hours, plates were spun down at $295 \times \mathrm{g}$ for 1 minute. Media was removed and cells were re-suspended in $30 \mu \mathrm{L}$ of Turbo Capture Lysis (TCL) buffer (Qiagen) containing 1\% beta-mercaptoethanol (BME). Finally, PLATE-Seq analyses was performed as described (Bush et al., 2017) at 24h after drug perturbation. Cells were harvested at 24h after perturbation with 335 compounds, including 117 FDA-approved oncology drugs and 218 experimental compounds (Table S5A). The screen was performed by the Columbia JP Sulzberger Genome Center High-Throughput Screening facility of the Herbert Irving Comprehensive Cancer Center (HICCC), which makes PLATE-seq screens available to the entire research community.

While the study used only profiles from DU145 cells - since they were a better model for the aggressive mCRPC samples in the SU2C cohort-drug perturbation profiles from both LNCaP and DU145 cells will be accessible from GEO. 
Vasciaveo et al.

Data analysis: PLATE-Seq profiles were mapped to the human reference genome version Grch38 using STAR aligner (Dobin et al., 2013). Variance stabilization, from the DESeq2 package for R (Love et al., 2014), was used to normalize the data from each plate. To correct for potential cross-plate batch effects, we used the function combat from the sva package for $\mathrm{R}$ (Leek et al., 2012) to compute the batch-corrected normalized gene expression and to fit a linear model for each compound against six plate-matched DMSO controls, included to minimize the need for cross-plate normalization. We used the limma package for $\mathrm{R}$ (Ritchie et al., 2015) to fit the linear model and to compute $P$-values and moderated t-statistics for each gene. For each compound, we used a vector of these statistics to generate a gene expression profile of z-scores representing the compound effect as differential between post- and pre-treatment. To account for tumor context-specificity, we performed VIPER-inference on the DU145 screening data using the SU2C interactome (as described above). The drug perturbation data are provided in Table S5B.

\section{OncoTreat Analysis}

OncoTreat analysis was performed as described in (Alvarez et al., 2018). Briefly, drugmediated MR inversion was independently assessed for each patient in the SU2C cohort and each GEMM as follows: for each sample and for each drug, we assessed the NES of the sample's MR-activity signature $(25 \uparrow+25 \downarrow$ most differentially active proteins) in proteins differentially inactivated and activated in drug-treated vs. vehicle control treated cells, respectively, using the aREA algorithm (Alvarez et al., 2016). NES values were then converted to P-values, and Bonferroni corrected to account for multiple hypothesis testing. An efficacy score was computed $S_{E}=-\log _{10} P$ and used to identify MR-inverter drugs $\left(S_{E} \geq 5\right.$, corresponding to $\left.P \leq 10^{-5}\right)$. Results of OncoTreat analyses for the SU2C and GEMM cohorts are provided in Table S5C and Table S5D, respectively. 
Vasciaveo et al.

\section{OncoLoop Algorithm}

The OncoLoop algorithm leverages a tripartite graph $T P G$ with nodes representing patients $\left(P_{i}\right)$, GEMM-DT $\left(G_{j}\right)$ and drugs $\left(D_{k}\right)$, respectively, and edges represent statistically significant GEMM-patient fidelity score $S_{M}\left(P_{i}, G_{j}\right) \geq 5$, GEMM-drug MR-inverter score $S_{E}\left(G_{j}, D_{k}\right) \geq 5$ and patient-drug MR-inverter score $S_{E}\left(P_{i}, D_{k}\right) \geq 5$. All closed 3-node loops including a patient, a GEMM-DT, and a drug are considered as statistically significant PGD-Loops. These were then ranked based on the Stouffer's integration of the z-scores corresponding to the $S_{M}\left(P_{i}, G_{j}\right)$, $S_{E}\left(G_{j}, D_{k}\right)$, and $S_{E}\left(P_{i}, D_{k}\right)$ values of the loop, which can then be converted back to a P-value.

Analysis of 212 human samples, 91 GEMM samples, and 335 drug perturbations yielded 668,138 statistically significant PGD-Loops, which are summarized in Table S6.

For each Patient, the optimal GEMM-DT for validation and most likely MR-inverter drug(s) for both patient and GEMM-DT can be identified by selecting among all the PGD-Loops that include that patient, those with the highest Stouffer's integrated rank.

\section{Preclinical Validation of OncoLoop-predicted Drugs}

Candidate drug selection: To select candidate drugs for in vivo validation we analyzed the 668,138 significant PGD-Loops produced by OncoLoop analysis of SU2C cohort patients, as described in the previous section. Specifically, to maximize the translational potential of selected drugs, we focused on loops restricted to (a) including only FDA-approved drugs; $(b)$ including drugs tested at a physiologically relevant concentration $\leq 1 \mu M$, and $(c)$ including drugs predicted as MR-inverters for $\geq 50 \%$ of SU2C samples $(n=212)$, thus suggesting broad applicability. The analysis yielded 16 drugs, which were then sorted by patient coverage-i.e., the fraction of patients in PGD-Loops comprising the specific drug (Figure 6A,B). 
Vasciaveo et al.

Candidate drug validation: For validation of candidate drugs, we tested their ability to inhibit tumor growth in vivo in cognate GEMMs. Cognate GEMMs for validation were selected based on their being (1) included in the closed loops (i.e., matching both the drug and the SU2C patient) and (2) readily available as allograft models. Based on these criteria, we selected two allograft models: NPp53 ${ }^{\text {mut }}$ CMZ315 and NPM CMZ150 (Table S1B). In addition, we validated drug candidates in the MR-matched human PDX LUCAP-73 model (Table S7B) (Nguyen et al., 2017). For the allograft tumors, $20-40 \mathrm{mg}$ pieces from passage 2 tumors were grown in the flanks of male NCr nude mice (Envigo), as above. For the LUCAP-73 xenografts, $100 \mathrm{mg}$ pieces were grown in $\mathrm{R} 2 \mathrm{G} 2^{\circledR}$ mice (B6;129-Rag2tm1Fwall2rgtm1Rsky/DwlHsd, Envigo). Tumors were monitored by caliper measurement twice weekly and tumor volumes were calculated using the formula [Volume $=(\text { width })^{2} \times$ length/2]. When tumors reached $100-200 \mathrm{~mm}^{3}$, mice with similar mean tumor volume were randomized into vehicle and treatment groups.

Pharmaceutical grade compounds for the 4 candidate drugs, namely Temsirolimus (S1044), Trametinib (S2673), Panobinostat (S1030), Bortezomib (S1013), and Cabazitaxel (S3022) as a control for standard of care, were purchased from Sellekchem (Houston, TX). Stock solutions were made by dissolving the compounds in $100 \%$ ethanol (Temsirolimus) or DMSO (all others) that were frozen as single-use aliquots at $-80^{\circ} \mathrm{C}$. Stock solutions of the drugs were freshly diluted before use in vehicle (5\%PEG400, 5\%Tween80 in PBS). For each candidate drug, the dosage, mode of delivery and schedule was chosen based on previous reports and were as follows: Temsirolimus (20mg/kg) (Yu et al., 2001), Trametinib (1 $\mathrm{mg} / \mathrm{kg}$ ) (Li et al., 2019), Panobinostat (15mg/kg) (Bruzzese et al., 2013), Bortezomib (1 $\mathrm{mg} / \mathrm{kg})$ (Garcia-Gomez et al., 2014), and Cabazitaxel (10mg/kg) (Vidal et al., 2015) Drugs were administered via intraperitoneal delivery (i.p.) 3 times/week in non-consecutive days (Temsirolimus, Panobinostat, Bortezomib, and Cabazitaxel), or by oral gavage 5 times/week in consecutive days (Trametinib). Tumors were 
harvested when the tumor size of vehicle treated mice reached $2 \mathrm{~cm}$ or earlier if the body condition score of the host mice were $<1.5$ or if they exhibited signs of distress. Growth curves were analyzed using two-way ANOVA with Dunnet's multiple comparisons test, compared to vehicletreated controls. Mice with tumors greater than $2.0 \mathrm{~cm}$, or having $20 \%$ weight loss from baseline, or with body condition scoring of 1.5 or less were euthanized by $\mathrm{CO} 2$ inhalation followed by cervical dislocation to verify death. Tumors were fixed in $10 \%$ formalin to be processed for histology, or snap-frozen in liquid nitrogen.

Pharmacokinetic analyses: To quantify the abundance of drug in the treated tumors, pharmacokinetic studies were performed using Ultra Performance Liquid ChromatographyTandem Mass Spectrometry (UPLC-MSMS) by the Biomarkers Core Laboratory at CUIMC. Drugs were extracted from $10 \mathrm{mg}$ snap-frozen tissues from tissue homogenates spiked with internal standards by liquid-liquid extraction using diethyl ether: ethyl acetate (30:70) and resuspended in $20 \%$ methanol for LCMS analysis. LCMS analysis was carried out on a Waters Xevo TQ MS integrated with ACQUITY UPLC system (Waters, Milford, MA, USA). The system was controlled by MassLynx Software 4.1. Single assays were used for bortezomib and Temsirolimus whereas Panobinostat and Trametinib were measured in two separate LCMS reactions. The lower limit of quantitation was $2.5 \mathrm{ng} / \mathrm{mL}$ (Trametinib, Borteozomib) and 5ng/mL (Temsirolimus, panobinostat). The intra-assay precision was as follows: Temsirolimus 5.34\%, panobinostat $5.06 \%$, Trametinib $4.83 \%$ and bortezomib, $3.20 \%$.

Pharmacodynamic analyses: Pharmacodynamic assays were performed at an early time point following in vivo drug treatment to assess each drug's ability to recapitulate the computationally predicted MR inversion. For this purpose, allografted tumors were grown in host mice, as above. When the tumors reached the size of $100-200 \mathrm{~mm}^{3}$, the host mice were treated with drug, as above, once daily for three consecutive days at the above-indicated doses. On the 
Vasciaveo et al.

morning of day 4, tumors were collected and snap-frozen for RNA extraction or fixed in formalin. RNA sequencing was performed as described above for GEMMs. The data is deposited in GEO (GSE186566).

RNA-seq profiles were pseudo-aligned to mouse reference genome version GRCm38 mm10, using kallisto v0.44.0 (Bray et al., 2016) with sequence-based bias correction. Transcript abundance was quantified and summarized in gene counts mapped to the ENTREZ gene model. We used limma voom to transform count data to log2-counts per million ( $\log _{2} \mathrm{CPM}$ ) and account for mean-variance relationship (Ritchie et al., 2015). Next, we fitted gene-wise linear models and computed moderated t-statistics using empirical Bayes. Contrasts were created between treated cells and cell treated with vehicle only. Sample biological replicates $(n=3)$ were used as blocking factor in the experimental design. To generate a gene expression signature (GES) of treatmentinduced transcriptional changes we mapped the differential gene expression data (i.e., FDRadjusted p-values and log-Fold Change scores) to a normal distribution. Analyses of treatmentinduced MR-reversal was performed as described above.

\section{Statistical analyses}

Statistical analysis was performed using GraphPad Prism software (Version 9.3.1) and Rstudio (0.99.902, R v4.0.2). Kaplan-Meier survival analysis was performed using two-tailed logrank test compared to the NP model. Comparison of frequencies was done using two-tailed Fisher's exact test or as described in figure legends. Subcutaneous tumor growth curves were analyzed using One-way ANOVA at the last time point compared to Vehicle treated tumors, adjusted for multiple comparisons with Dunnett's test. All bars show the mean and error bars SD. GSEA were computed by custom code implemented as per (Subramanian et al., 2005) algorithm. $P$-values estimated using a null distribution of 1,000 permutations of random fixed-size gene sets; 
Vasciaveo et al.

NES, normalized enrichment score. No statistical method was used to predetermine the sample size used for in vivo experiments.

\section{Data Accession}

The following datasets are deposited in GEO: (a) The mouse gene expression profiles (raw and normalized data) (GSE186566) (b) The human PDX gene expression profiles (raw and normalized data) (GSE184427), and (c) The PLATE-seq data for the drug perturbation profiles in both LNCaP and DU145 cells. 


\section{Author Contributions:}

A.V. Conceptualized, designed and performed the computational analyses, and wrote manuscript.

M.Z. Conceptualized, designed and performed the generation and analysis of the GEMMs.

J.M.A. Conceptualized, designed and performed the drug validation studies, and wrote manuscript.

F.N.A. Designed and executed experiments, and performed analysis of the GEMMs.

E.F.D., C.K., R.R., and S.P. Designed and executed experiments, and performed data analysis related to the drug perturbation studies.

M.S., A.M., and C.W.C. Designed and executed experiments, and performed analysis of the PDX interactome.

A.R.C. and S.d.B. Performed histopathological analysis.

J.Y.K. Performed experiments.

E.C. Provided the PDX models

M.A. Performed data analysis.

M.M.S. and M.A.R. Supervisory role and data analysis.

A.C. Conceived the study, designed experiments, performed data analysis, and wrote manuscript.

C.A.S. Conceived the study, designed experiments, performed data analysis, and wrote manuscript. 
Vasciaveo et al.

Acknowledgements: We are grateful to Sarah Bergen, for assistance with PDX models, and Stephanie Afari for assistance with the GEMMs. Figure 1 was created with BioRender.com using an institutional license sponsored by Columbia University's VP\&S Office for Research.

These studies were supported by Flow Cytometry, Genomics and High Throughput Screening, and Oncology Precision Therapeutics and Imaging Core facilities which are funded in part through Herbert Irving Comprehensive Cancer Center support Grant P30 CA013696, the Biomarkers Core Laboratory at the Irving Institute for Clinical and Translational Research, home to Columbia University's Clinical and Translational Science Award UL1TR001873, the Translational Research Unit, Institute of Pathology, University of Bern, and PDX core at the University of Washington is supported by the PNW Prostate Cancer SPORE P50CA097186, and P01CA163227. Funding to support the deposition of mouse strains at The Jackson Laboratories was provided by the TJ Martell Foundation for Leukemia, Cancer and AIDS Research and the Prostate Cancer Foundation

This work was supported by the NCl Cancer Target Discovery and Development Program (U01 CA217858, to AC), the Cancer Systems Biology Consortium (U54 CA209997, to AC), NIH Shared Instrumentation Grants (S10 OD012351 and S1 0OD021764 to AC), R01 CA173481 (to CAS), R01 CA183929 (to CAS), P01 CA265768R01 (to MMS), CA238005 (to MMS), U01CA261822 (to MMS), and a Prostate Cancer Challenge Award (to MMS and AC). AV is supported by the U.S. Department of Defense Early Investigator Research Award (W81XWH19-1-0337) and an Early Career Development Pilot Award NIH/NCl Cancer Center funded through the Cancer Center Support grant, P30CA013696. MZ was supported in part by the National Center for Advancing Translational Sciences, National Institutes of Health, Grant Number UL1TR001873. JMA was supported by the Dean's Precision Medicine Research Fellowship from the Irving Institute for Clinical and Translational Research at Columbia University Irving Medical Center 
(UL1TR001873), and a Prostate Cancer Foundation Young Investigator Award.MS was supported by NIH K99/R00CA194287. AM was supported by a Prostate Cancer Foundation (PCF) Young Investigator award. C.A.S. is an American Cancer Society Research Professor supported in part by a generous gift from the F.M. Kirby Foundation.

\section{Conflict of interest to report:}

A.C. is founder, equity holder, and consultant of DarwinHealth Inc., a company that has licensed some of the algorithms used in this manuscript from Columbia University. Columbia University is also an equity holder in DarwinHealth Inc.

M.J.A. is CSO and equity holder of DarwinHealth, Inc. Columbia University is also an equity holder in DarwinHealth.

E.C. received research funding under institutional SRA from Janssen Research and Development, Bayer Pharmaceuticals, KronosBio, Forma Pharmaceutics Foghorn, Gilead, Sanofi, AbbVie, and GSK. 
Vasciaveo et al.

\section{References}

Abida, W., Cyrta, J., Heller, G., Prandi, D., Armenia, J., Coleman, I., Cieslik, M., Benelli, M., Robinson, D., Van Allen, E.M., et al. (2019). Genomic correlates of clinical outcome in advanced prostate cancer. Proc Natl Acad Sci U S A 116, 11428-11436.

Alvarez, M.J., Shen, Y., Giorgi, F.M., Lachmann, A., Ding, B.B., Ye, B.H., and Califano, A. (2016). Functional characterization of somatic mutations in cancer using network-based inference of protein activity. Nat Genet $48,838-847$.

Alvarez, M.J., Subramaniam, P.S., Tang, L.H., Grunn, A., Aburi, M., Rieckhof, G., Komissarova, E.V., Hagan, E.A., Bodei, L., Clemons, P.A., et al. (2018). A precision oncology approach to the pharmacological targeting of mechanistic dependencies in neuroendocrine tumors. Nat Genet 50, 979-989.

Alvarez, M.J., Yan, P., Alpaugh, M.L., Bowden, M., Sicinska, E., Zhou, C.W., Karan, C., Realubit, R.B., Mundi, P.S., Grunn, A., et al. (2019). Reply to 'H-STS, L-STS and KRJ-I are not authentic GEPNET cell lines'. Nat Genet 51, 1427-1428.

Armenia, J., Wankowicz, S.A.M., Liu, D., Gao, J., Kundra, R., Reznik, E., Chatila, W.K., Chakravarty, D., Han, G.C., Coleman, I., et al. (2018). The long tail of oncogenic drivers in prostate cancer. Nat Genet 50, 645-651.

Arora, V.K., Schenkein, E., Murali, R., Subudhi, S.K., Wongvipat, J., Balbas, M.D., Shah, N., Cai, L., Efstathiou, E., Logothetis, C., et al. (2013). Glucocorticoid receptor confers resistance to antiandrogens by bypassing androgen receptor blockade. Cell 155, 1309-1322.

Arriaga, J.M., and Abate-Shen, C. (2019). Genetically Engineered Mouse Models of Prostate Cancer in the Postgenomic Era. Cold Spring Harb Perspect Med 9.

Arriaga, J.M., Panja, S., Alshalalfa, M., Zhao, J., Zou, M., Giacobbe, A., Madubata, C.J., Yeji Kim, J., Rodriguez, A., Coleman, I., et al. (2020). A MYC and RAS co-activation signature in localized prostate cancer drives bone metastasis and castration resistance. Nat Cancer 1 , 1082-1096.

Ashburner, M., Ball, C.A., Blake, J.A., Botstein, D., Butler, H., Cherry, J.M., Davis, A.P., Dolinski, K., Dwight, S.S., Eppig, J.T., et al. (2000). Gene ontology: tool for the unification of biology. The Gene Ontology Consortium. Nat Genet 25, 25-29.

Aytes, A., Giacobbe, A., Mitrofanova, A., Ruggero, K., Cyrta, J., Arriaga, J., Palomero, L., FarranMatas, S., Rubin, M.A., Shen, M.M., et al. (2018). NSD2 is a conserved driver of metastatic prostate cancer progression. Nat Commun 9, 5201.

Aytes, A., Mitrofanova, A., Kinkade, C.W., Lefebvre, C., Lei, M., Phelan, V., LeKaye, H.C., Koutcher, J.A., Cardiff, R.D., Califano, A., et al. (2013). ETV4 promotes metastasis in response to activation of PI3-kinase and Ras signaling in a mouse model of advanced prostate cancer. Proc Natl Acad Sci U S A 110, E3506-3515.

Aytes, A., Mitrofanova, A., Lefebvre, C., Alvarez, M.J., Castillo-Martin, M., Zheng, T., Eastham, J.A., Gopalan, A., Pienta, K.J., Shen, M.M., et al. (2014). Cross-species regulatory network analysis identifies a synergistic interaction between FOXM1 and CENPF that drives prostate cancer malignancy. Cancer Cell 25, 638-651. 
Baca, S.C., Takeda, D.Y., Seo, J.H., Hwang, J., Ku, S.Y., Arafeh, R., Arnoff, T., Agarwal, S., Bell, C., O'Connor, E., et al. (2021). Reprogramming of the FOXA1 cistrome in treatmentemergent neuroendocrine prostate cancer. Nat Commun 12, 1979.

Basso, K., Margolin, A.A., Stolovitzky, G., Klein, U., Dalla-Favera, R., and Califano, A. (2005). Reverse engineering of regulatory networks in human B cells. Nat Genet 37, 382-390.

Bekes, M., Langley, D.R., and Crews, C.M. (2022). PROTAC targeted protein degraders: the past is prologue. Nat Rev Drug Discov.

Beltran, H., Prandi, D., Mosquera, J.M., Benelli, M., Puca, L., Cyrta, J., Marotz, C., Giannopoulou, E., Chakravarthi, B.V., Varambally, S., et al. (2016). Divergent clonal evolution of castrationresistant neuroendocrine prostate cancer. Nat Med 22, 298-305.

Bleijs, M., van de Wetering, M., Clevers, H., and Drost, J. (2019). Xenograft and organoid model systems in cancer research. EMBO J 38, e101654.

Blondel, V.D., Guillaume, J.-L., Lambiotte, R., and Lefebvre, E. (2008). Fast unfolding of communities in large networks. Journal of statistical mechanics: theory and experiment 2008, P10008.

Bray, N.L., Pimentel, H., Melsted, P., and Pachter, L. (2016). Near-optimal probabilistic RNA-seq quantification. Nat Biotechnol 34, 525-527.

Brichta, L., Shin, W., Jackson-Lewis, V., Blesa, J., Yap, E.L., Walker, Z., Zhang, J., Roussarie, J.P., Alvarez, M.J., Califano, A., et al. (2015). Identification of neurodegenerative factors using translatome-regulatory network analysis. Nat Neurosci 18, 1325-1333.

Bruzzese, F., Pucci, B., Milone, M.R., Ciardiello, C., Franco, R., Chianese, M.I., Rocco, M., Di Gennaro, E., Leone, A., Luciano, A., et al. (2013). Panobinostat synergizes with zoledronic acid in prostate cancer and multiple myeloma models by increasing ROS and modulating mevalonate and p38-MAPK pathways. Cell Death Dis 4, e878.

Bush, E.C., Ray, F., Alvarez, M.J., Realubit, R., Li, H., Karan, C., Califano, A., and Sims, P.A. (2017). PLATE-Seq for genome-wide regulatory network analysis of high-throughput screens. Nature communications 8, 105.

Cancer Genome Atlas Research, N. (2015). The Molecular Taxonomy of Primary Prostate Cancer. Cell 163, 1011-1025.

Chen, Y., Chi, P., Rockowitz, S., laquinta, P.J., Shamu, T., Shukla, S., Gao, D., Sirota, I., Carver, B.S., Wongvipat, J., et al. (2013). ETS factors reprogram the androgen receptor cistrome and prime prostate tumorigenesis in response to PTEN loss. Nat Med 19, 1023-1029.

Chua, C.W., Shibata, M., Lei, M., Toivanen, R., Barlow, L.J., Bergren, S.K., Badani, K.K., McKiernan, J.M., Benson, M.C., Hibshoosh, H., et al. (2014). Single luminal epithelial progenitors can generate prostate organoids in culture. Nat Cell Biol 16, 951-961, 951-954.

Chung, N.C., and Storey, J.D. (2015). Statistical significance of variables driving systematic variation in high-dimensional data. Bioinformatics 31, 545-554.

Colaprico, A., Silva, T.C., Olsen, C., Garofano, L., Cava, C., Garolini, D., Sabedot, T.S., Malta, T.M., Pagnotta, S.M., Castiglioni, I., et al. (2016). TCGAbiolinks: an R/Bioconductor package for integrative analysis of TCGA data. Nucleic Acids Res 44, e71.

Consortium, G.T. (2013). The Genotype-Tissue Expression (GTEx) project. Nat Genet 45, 580585. 
Crawford, E.D., Higano, C.S., Shore, N.D., Hussain, M., and Petrylak, D.P. (2015). Treating Patients with Metastatic Castration Resistant Prostate Cancer: A Comprehensive Review of Available Therapies. J Urol 194, 1537-1547.

Crooke, S.T., Witztum, J.L., Bennett, C.F., and Baker, B.F. (2018). RNA-Targeted Therapeutics. Cell Metab 27, 714-739.

Dankort, D., Filenova, E., Collado, M., Serrano, M., Jones, K., and McMahon, M. (2007). A new mouse model to explore the initiation, progression, and therapy of BRAFV600E-induced lung tumors. Genes Dev 21, 379-384.

Ding, H., Burgenske, D.M., Zhao, W., Subramaniam, P.S., Bakken, K.K., He, L., Alvarez, M.J., Laise, P., Paull, E.O., Spinazzi, E.F., et al. (2019). Single-cell based elucidation of molecularly-distinct glioblastoma states and drug sensitivity. https://doiorg/101101/675439.

Ding, H., Douglass, E.F., Jr., Sonabend, A.M., Mela, A., Bose, S., Gonzalez, C., Canoll, P.D., Sims, P.A., Alvarez, M.J., and Califano, A. (2018). Quantitative assessment of protein activity in orphan tissues and single cells using the metaVIPER algorithm. Nat Commun 9, 1471.

Dobin, A., Davis, C.A., Schlesinger, F., Drenkow, J., Zaleski, C., Jha, S., Batut, P., Chaisson, M., and Gingeras, T.R. (2013). STAR: ultrafast universal RNA-seq aligner. Bioinformatics 29, 15-21.

Douglass, E.F., Jr., Allaway, R.J., Szalai, B., Wang, W., Tian, T., Fernandez-Torras, A., Realubit, R., Karan, C., Zheng, S., Pessia, A., et al. (2022). A community challenge for a pancancer drug mechanism of action inference from perturbational profile data. Cell Rep Med 3, 100492.

Echeverria, G.V., Ge, Z., Seth, S., Zhang, X., Jeter-Jones, S., Zhou, X., Cai, S., Tu, Y., McCoy, A., Peoples, M., et al. (2019). Resistance to neoadjuvant chemotherapy in triple-negative breast cancer mediated by a reversible drug-tolerant state. Sci Transl Med 11.

Ellwood-Yen, K., Graeber, T.G., Wongvipat, J., Iruela-Arispe, M.L., Zhang, J., Matusik, R., Thomas, G.V., and Sawyers, C.L. (2003). Myc-driven murine prostate cancer shares molecular features with human prostate tumors. Cancer Cell 4, 223-238.

Fabregat, A., Jupe, S., Matthews, L., Sidiropoulos, K., Gillespie, M., Garapati, P., Haw, R., Jassal, B., Korninger, F., May, B., et al. (2018). The Reactome Pathway Knowledgebase. Nucleic Acids Res 46, D649-D655.

Floc'h, N., Kinkade, C.W., Kobayashi, T., Aytes, A., Lefebvre, C., Mitrofanova, A., Cardiff, R.D., Califano, A., Shen, M.M., and Abate-Shen, C. (2012). Dual targeting of the Akt/mTOR signaling pathway inhibits castration-resistant prostate cancer in a genetically engineered mouse model. Cancer Res 72, 4483-4493.

Gao, D., Vela, I., Sboner, A., laquinta, P.J., Karthaus, W.R., Gopalan, A., Dowling, C., Wanjala, J.N., Undvall, E.A., Arora, V.K., et al. (2014). Organoid cultures derived from patients with advanced prostate cancer. Cell 159, 176-187.

Garcia-Gomez, A., Quwaider, D., Canavese, M., Ocio, E.M., Tian, Z., Blanco, J.F., Berger, A.J., Ortiz-de-Solorzano, C., Hernandez-Iglesias, T., Martens, A.C., et al. (2014). Preclinical activity of the oral proteasome inhibitor MLN9708 in Myeloma bone disease. Clin Cancer Res 20, 1542-1554. 
Houle, M.E., Kriegel, H.-P., Kröger, P., Schubert, E., and Zimek, A. (2010). Can Shared-Neighbor Distances Defeat the Curse of Dimensionality? (Berlin, Heidelberg: Springer Berlin Heidelberg).

Jackson, E.L., Willis, N., Mercer, K., Bronson, R.T., Crowley, D., Montoya, R., Jacks, T., and Tuveson, D.A. (2001). Analysis of lung tumor initiation and progression using conditional expression of oncogenic K-ras. Genes Dev 15, 3243-3248.

Kanehisa, M., and Goto, S. (2000). KEGG: kyoto encyclopedia of genes and genomes. Nucleic Acids Res 28, 27-30.

Kersten, K., de Visser, K.E., van Miltenburg, M.H., and Jonkers, J. (2017). Genetically engineered mouse models in oncology research and cancer medicine. EMBO Mol Med 9, 137-153.

Kruczek, K., Ratterman, M., Tolzien, K., Sulo, S., Lestingi, T.M., and Nabhan, C. (2013). A phase II study evaluating the toxicity and efficacy of single-agent temsirolimus in chemotherapynaive castration-resistant prostate cancer. Br J Cancer 109, 1711-1716.

Lachmann, A., Giorgi, F.M., Lopez, G., and Califano, A. (2016). ARACNe-AP: gene network reverse engineering through adaptive partitioning inference of mutual information. Bioinformatics 32, 2233-2235.

Leek, J.T., Johnson, W.E., Parker, H.S., Jaffe, A.E., and Storey, J.D. (2012). The sva package for removing batch effects and other unwanted variation in high-throughput experiments. Bioinformatics 28, 882-883.

Lesche, R., Groszer, M., Gao, J., Wang, Y., Messing, A., Sun, H., Liu, X., and Wu, H. (2002). Cre/loxP-mediated inactivation of the murine Pten tumor suppressor gene. Genesis 32, 148149.

Li, S., Fong, K.W., Gritsina, G., Zhang, A., Zhao, J.C., Kim, J., Sharp, A., Yuan, W., Aversa, C., Yang, X.J., et al. (2019). Activation of MAPK Signaling by CXCR7 Leads to Enzalutamide Resistance in Prostate Cancer. Cancer Res 79, 2580-2592.

Liberzon, A., Birger, C., Thorvaldsdottir, H., Ghandi, M., Mesirov, J.P., and Tamayo, P. (2015). The Molecular Signatures Database (MSigDB) hallmark gene set collection. Cell Syst 1, 417-425.

Logothetis, C., Morris, M.J., Den, R., and Coleman, R.E. (2018). Current perspectives on bone metastases in castrate-resistant prostate cancer. Cancer Metastasis Rev 37, 189-196.

Love, M.I., Huber, W., and Anders, S. (2014). Moderated estimation of fold change and dispersion for RNA-seq data with DESeq2. Genome Biol 15, 550.

Ma, J., Fong, S.H., Luo, Y., Bakkenist, C.J., Shen, J.P., Mourragui, S., Wessels, L.F.A., Hafner, M., Sharan, R., Peng, J., et al. (2021). Few-shot learning creates predictive models of drug response that translate from high-throughput screens to individual patients. Nat Cancer 2, 233-244.

Madisen, L., Zwingman, T.A., Sunkin, S.M., Oh, S.W., Zariwala, H.A., Gu, H., Ng, L.L., Palmiter, R.D., Hawrylycz, M.J., Jones, A.R., et al. (2010). A robust and high-throughput Cre reporting and characterization system for the whole mouse brain. Nat Neurosci 13, 133-140.

Margolin, A.A., Wang, K., Lim, W.K., Kustagi, M., Nemenman, I., and Califano, A. (2006). Reverse engineering cellular networks. Nature protocols 1, 662-671. 
Vasciaveo et al.

Mattar, M., McCarthy, C.R., Kulick, A.R., Qeriqi, B., Guzman, S., and de Stanchina, E. (2018). Establishing and Maintaining an Extensive Library of Patient-Derived Xenograft Models. Front Oncol 8, 19.

Mclnnes, L., Healy, J., and Melville, J. (2018). Umap: Uniform manifold approximation and projection for dimension reduction. arXiv preprint arXiv:180203426.

Mishra, V., Re, D.B., Le Verche, V., Alvarez, M.J., Vasciaveo, A., Jacquier, A., Doulias, P.T., Greco, T.M., Nizzardo, M., Papadimitriou, D., et al. (2020). Systematic elucidation of neuronastrocyte interaction in models of amyotrophic lateral sclerosis using multi-modal integrated bioinformatics workflow. Nat Commun 11, 5579.

Nguyen, H.M., Vessella, R.L., Morrissey, C., Brown, L.G., Coleman, I.M., Higano, C.S., Mostaghel, E.A., Zhang, X., True, L.D., Lam, H.M., et al. (2017). LuCaP Prostate Cancer Patient-Derived Xenografts Reflect the Molecular Heterogeneity of Advanced Disease an-d Serve as Models for Evaluating Cancer Therapeutics. Prostate 77, 654-671.

Nuhn, P., De Bono, J.S., Fizazi, K., Freedland, S.J., Grilli, M., Kantoff, P.W., Sonpavde, G., Sternberg, C.N., Yegnasubramanian, S., and Antonarakis, E.S. (2019). Update on Systemic Prostate Cancer Therapies: Management of Metastatic Castration-resistant Prostate Cancer in the Era of Precision Oncology. Eur Urol 75, 88-99.

Obradovic, A., Chowdhury, N., Haake, S.M., Ager, C., Wang, V., Vlahos, L., Guo, X.V., Aggen, D.H., Rathmell, W.K., Jonasch, E., et al. (2021). Single-cell protein activity analysis identifies recurrence-associated renal tumor macrophages. Cell 184, 2988-3005 e2916.

Olive, K.P., Tuveson, D.A., Ruhe, Z.C., Yin, B., Willis, N.A., Bronson, R.T., Crowley, D., and Jacks, T. (2004). Mutant p53 gain of function in two mouse models of Li-Fraumeni syndrome. Cell 119, 847-860.

Paull, E.O., Aytes, A., Jones, S.J., Subramaniam, P.S., Giorgi, F.M., Douglass, E.F., Tagore, S., Chu, B., Vasciaveo, A., Zheng, S., et al. (2021). A modular master regulator landscape controls cancer transcriptional identity. Cell 184, 334-351 e320.

Puca, L., Bareja, R., Prandi, D., Shaw, R., Benelli, M., Karthaus, W.R., Hess, J., Sigouros, M., Donoghue, A., Kossai, M., et al. (2018). Patient derived organoids to model rare prostate cancer phenotypes. Nat Commun 9, 2404.

Rathkopf, D.E., Picus, J., Hussain, A., Ellard, S., Chi, K.N., Nydam, T., Allen-Freda, E., Mishra, K.K., Porro, M.G., Scher, H.I., et al. (2013). A phase 2 study of intravenous panobinostat in patients with castration-resistant prostate cancer. Cancer Chemother Pharmacol 72, 537544.

Repunte-Canonigo, V., Shin, W., Vendruscolo, L.F., Lefebvre, C., van der Stap, L., Kawamura, T., Schlosburg, J.E., Alvarez, M., Koob, G.F., Califano, A., et al. (2015). Identifying candidate drivers of alcohol dependence-induced excessive drinking by assembly and interrogation of brain-specific regulatory networks. Genome Biol 16, 68.

Rickman, D.S., Beltran, H., Demichelis, F., and Rubin, M.A. (2017). Biology and evolution of poorly differentiated neuroendocrine tumors. Nat Med 23, 1-10.

Risom, T., Langer, E.M., Chapman, M.P., Rantala, J., Fields, A.J., Boniface, C., Alvarez, M.J., Kendsersky, N.D., Pelz, C.R., Johnson-Camacho, K., et al. (2018). Differentiation-state plasticity is a targetable resistance mechanism in basal-like breast cancer. Nat Commun 9 , 3815. 
Ritchie, M.E., Phipson, B., Wu, D., Hu, Y., Law, C.W., Shi, W., and Smyth, G.K. (2015). limma powers differential expression analyses for RNA-sequencing and microarray studies. Nucleic Acids Res 43, e47.

Rodon, J., Soria, J.C., Berger, R., Miller, W.H., Rubin, E., Kugel, A., Tsimberidou, A., Saintigny, P., Ackerstein, A., Brana, I., et al. (2019). Genomic and transcriptomic profiling expands precision cancer medicine: the WINTHER trial. Nat Med 25, 751-758.

Rousseeuw, P.J. (1987). Silhouettes - a Graphical Aid to the Interpretation and Validation of Cluster-Analysis. J Comput Appl Math 20, 53-65.

Sartor, O., and de Bono, J.S. (2018). Metastatic Prostate Cancer. N Engl J Med 378, 645-657.

Scher, H.I., and Sawyers, C.L. (2005). Biology of progressive, castration-resistant prostate cancer: directed therapies targeting the androgen-receptor signaling axis. J Clin Oncol 23, 8253-8261.

Schutgens, F., and Clevers, H. (2020). Human Organoids: Tools for Understanding Biology and Treating Diseases. Annu Rev Pathol 15, 211-234.

Shen, M.M., and Abate-Shen, C. (2010). Molecular genetics of prostate cancer: new prospects for old challenges. Genes Dev 24, 1967-2000.

Siegel, R.L., Miller, K.D., Fuchs, H.E., and Jemal, A. (2021). Cancer Statistics, 2021. CA Cancer J Clin 71, 7-33.

Smedley, D., Haider, S., Durinck, S., Pandini, L., Provero, P., Allen, J., Arnaiz, O., Awedh, M.H., Baldock, R., Barbiera, G., et al. (2015). The BioMart community portal: an innovative alternative to large, centralized data repositories. Nucleic Acids Res 43, W589-598.

Son, J., Ding, H., Farb, T.B., Efanov, A.M., Sun, J., Gore, J.L., Syed, S.K., Lei, Z., Wang, Q., Accili, D., et al. (2021). BACH2 inhibition reverses beta cell failure in type 2 diabetes models. J Clin Invest 131.

Stuart, T., Butler, A., Hoffman, P., Hafemeister, C., Papalexi, E., Mauck, W.M., 3rd, Hao, Y., Stoeckius, M., Smibert, P., and Satija, R. (2019). Comprehensive Integration of Single-Cell Data. Cell 177, 1888-1902 e1821.

Subramanian, A., Tamayo, P., Mootha, V.K., Mukherjee, S., Ebert, B.L., Gillette, M.A., Paulovich, A., Pomeroy, S.L., Golub, T.R., Lander, E.S., et al. (2005). Gene set enrichment analysis: a knowledge-based approach for interpreting genome-wide expression profiles. Proc Natl Acad Sci U S A 102, 15545-15550.

Tentler, J.J., Tan, A.C., Weekes, C.D., Jimeno, A., Leong, S., Pitts, T.M., Arcaroli, J.J., Messersmith, W.A., and Eckhardt, S.G. (2012). Patient-derived tumour xenografts as models for oncology drug development. Nat Rev Clin Oncol 9, 338-350.

Trapnell, C., Pachter, L., and Salzberg, S.L. (2009). TopHat: discovering splice junctions with RNA-Seq. Bioinformatics 25, 1105-1111.

Trapnell, C., Williams, B.A., Pertea, G., Mortazavi, A., Kwan, G., van Baren, M.J., Salzberg, S.L., Wold, B.J., and Pachter, L. (2010). Transcript assembly and quantification by RNA-Seq reveals unannotated transcripts and isoform switching during cell differentiation. Nat Biotechnol 28, 511-515. 
Vidal, S.J., Rodriguez-Bravo, V., Quinn, S.A., Rodriguez-Barrueco, R., Lujambio, A., Williams, E., Sun, X., de la Iglesia-Vicente, J., Lee, A., Readhead, B., et al. (2015). A targetable GATA2IGF2 axis confers aggressiveness in lethal prostate cancer. Cancer Cell 27, 223-239.

Wang, J., Kobayashi, T., Floc'h, N., Kinkade, C.W., Aytes, A., Dankort, D., Lefebvre, C., Mitrofanova, A., Cardiff, R.D., McMahon, M., et al. (2012). B-Raf activation cooperates with PTEN loss to drive c-Myc expression in advanced prostate cancer. Cancer Res 72, 47654776.

Wang, X., Kruithof-de Julio, M., Economides, K.D., Walker, D., Yu, H., Halili, M.V., Hu, Y.P., Price, S.M., Abate-Shen, C., and Shen, M.M. (2009). A luminal epithelial stem cell that is a cell of origin for prostate cancer. Nature 461, 495-500.

Warren, A., Chen, Y., Jones, A., Shibue, T., Hahn, W.C., Boehm, J.S., Vazquez, F., Tsherniak, A., and McFarland, J.M. (2021). Global computational alignment of tumor and cell line transcriptional profiles. Nat Commun 12, 22.

Watson, P.A., Arora, V.K., and Sawyers, C.L. (2015). Emerging mechanisms of resistance to androgen receptor inhibitors in prostate cancer. Nature reviews Cancer 15, 701-711.

Yu, K., Toral-Barza, L., Discafani, C., Zhang, W.G., Skotnicki, J., Frost, P., and Gibbons, J.J. (2001). mTOR, a novel target in breast cancer: the effect of CCI-779, an mTOR inhibitor, in preclinical models of breast cancer. Endocr Relat Cancer 8, 249-258.

Zou, M., Toivanen, R., Mitrofanova, A., Floch, N., Hayati, S., Sun, Y., Le Magnen, C., Chester, D., Mostaghel, E.A., Califano, A., et al. (2017). Transdifferentiation as a Mechanism of Treatment Resistance in a Mouse Model of Castration-Resistant Prostate Cancer. Cancer Discov 7, 736-749. 
Vasciaveo et al.

\section{Figure Legends}

\section{Figure 1: The OncoLoop conceptual framework}

A. Conceptual overview: OncoLoop was designed to identify high-fidelity (cognate) models of a patient tumor-i.e., GEMM-derived tumors (GEMM-DTs), in this study-as well as drugs capable of inverting the activity of MR proteins identified from both the patient and his cognate GEMM-DT samples for follow-up, patient-relevant validation. To accomplish this goal, OncoLoop performs integrative analysis of transcriptomic (RNA-seq) profiles from a patient tumor, his cognate model, and large-scale drug perturbation assays. B. Regulatory network analysis: Gene expression profiles generated from each data source were used to reverse-engineer species- and cohortspecific regulatory networks, which are then used to transform differential gene expression signature from individual samples into differential protein activity profiles. Drug perturbation profiles were analyzed with patient-derived networks (See Methods). C. OncoLoop analysis: Gene Set Enrichment Analysis, as implemented by the aREA algorithm (Alvarez et al., 2016), was used to assess the overlap in differentially active MR proteins between a human tumor and its cognate GEMM-DTs (OncoMatch algorithm). Similarly, aREA-based enrichment analysis was used to identify drugs capable of inverting the activity of a tumor's MRs in drug- vs. vehicle control treated cells (MR-inverter drugs), for each patient and cognate GEMM-DT(s) pairs. D. Drug prediction and validation: Representative Circos plot illustrating PGD-loops generated by matching a patient $(P)$ to a GEMM-DT $(G)$ by identifying the same MR-inverter drug $(D)$ for both. Candidate drugs were first prioritized by pharmacotype analysis (i.e., subset of patients/GEMMDTs predicted to be sensitive to the same subset of drugs) and then validated in vivo using, both in cognate GEMM-DT-derived allografts as well as in MR-matched PDX models. 
Vasciaveo et al.

\section{Figure 2: A GEMM resource that models prostate cancer progression}

A. Strategy: Schematic showing representative GEMMs used in this study. The GEMM cohort

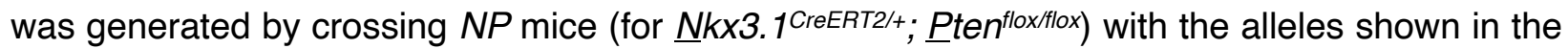
panel to generate six complex strains (see Table 1 for details). A timeline for tumor induction, castration, monitoring, and sacrificing is shown at the bottom of the panel. B. Castrationresistance: $\mathrm{MRI}$ images showing tumor volume changes after castration of two representative NPp53 mice. The plot on the right shows the tumor volume changes over time for 4 representative NPp53 mice. C. Metastatic Progression: Frequency of metastasis observed in the GEMMs. The numbers of mice used to determine the metastasis frequency in each model are indicated in parentheses; two-tailed $P$-values are shown for Fisher's exact test comparing each model to the NP mice (as a control). OG stands for outcome group. D. Kaplan-Meier analysis: analysis of GEMMs' survival curves identified three outcome groups $\left(\mathrm{OG}_{1}, \mathrm{OG}_{2}\right.$, and $\left.\mathrm{OG}_{3}\right)$. $P$-values are shown for two-tailed log-rank test compared to the NP mice. For the analyses shown in (C) and (D), both intact and castrated mice were pooled for all GEMMs except NPM, where the effects of castration may be confounded by the AR-dependency of the Probasin promoter used to drive Myc expression (see Methods). E. Histopathology: Representative images for hematoxylin and eosin (H\&E) (top row) and immunohistochemical staining of the indicated markers in primary tumors of the different GEMMs. Shown are representative images for 3 mice; scale bars represent 50um. See also Table S1, and Figures S1-S3.

\section{Figure 3: GEMM subtypes recapitulate human PCa phenotypes}

A. Cluster analysis: Heatmap illustrating the results of protein activity-based cluster analysis of $\mathrm{n}=91$ GEMM-derived tumors (GEMM-DTs), as well as the silhouette score and correlative variables, such as survival group, castration status, and metastatic progression, see STAR Methods. The analysis identified five molecularly-distinct clusters $\left(C_{1}-C_{5}\right)$ that co-segregate with 
Vasciaveo et al.

survival and metastatic potential. For each cluster, we report the 10 most significantly activated MRs (top heatmap); we also report selected, established human PCa markers (bottom heatmap), whose activity is extremely well aligned with the 5-cluster solution. B. Representative subnetworks, representing the activity of the 25 most differentially active MR proteins (5/cluster, large circles) across all clusters the and expression of their regulatory targets (small circles) on a cluster-by-cluster basis. These networks illustrate the dramatic changes in downstream transcriptional programs that differentiate the biology of the corresponding GEMM-DTs. Protein activity is shown using a blue (inactivated) to red(activated) scale, while target expression is shown on a blue (under-expressed) to yellow (over-expressed) scale. High resolution images, with full visibility of the MR identities is shown in Supplementary Figure S5. See also Table S2, and Figures S4 and S5.

\section{Figure 4: Matching GEMM-DTs to patient tumor and metastases}

A. Human to GEMM-DT MR conservation: Heatmaps showing the MR-based fidelity score of each patient (columns) vs. each available GEMM-DT model (rows), as assessed by MR-activity conservation for TCGA (right) and SU2C patients (left), respectively, see STAR Methods and Tables S4A,B. Relevant patient phenotypic variables-i.e., Cohort Id, Gleason score and NEPC status - are shown in the top three bars, while relevant GEMM-DT phenotypic variables-i.e., Cluster Id, outcome, castration, and metastasis status - are shown in the four vertical bars to the left of the heatmap, see legend at the bottom of the panel. The top 5 cognate models of each patient are shown in dark red. Other statistically significant $\left(P \leq 10^{-5}\right)$ high-fidelity models are shown using a light to dark color scale. Fidelity scores are computed as the $-\log _{10} P$ of the patient vs. GEMM-DT MR enrichment analysis. The light blue barplots at the bottom of the two heatmaps shows the number of candidate cognate models for each patient, while the dark blue barplots to the right shows the number of patients for which a GEMM-DT represents a cognate model. 
Notably, the best cognate model for a give patient typically has much a more significant p-value, compared to the actual threshold, see panel B. Overall, $78 \%$ of the samples in the TCGA $(n=261$ of 334$)$ and $93 \%$ of those in the SU2C cohorts ( $n=198$ of 212 ) have at least one cognate GEMMDT. B. GSEA analysis: GSEA representation of the fidelity analysis for a low- (top) vs. high-fidelity (bottom) GEMM-DT models of two SU2C samples.

\section{Figure 5: OncoLoop analysis}

A. PGD-Loop identification: Illustrative examples of how PGD-Loops are identified, using three heatmaps representing a small, yet representative subset of patients, GEMM-DTs, and drugs. The top left heatmap (fidelity analysis) shows the fidelity score results for 56 SU2C samples (columns) and 5 GEMM-DTs (rows). The bottom left heatmap shows the MR-inverter scores for 28 drugs (rows) as assessed against 56 SU2C samples (columns), while the top right heatmap shows the MR-inverter scores for the same 28 drugs (columns) as assessed against the 5 GEMMDTs (rows). See Table S6 for the full set of scores across all patients, GEMM-DTs, and drugs. All scores are computed as $\left(-\log _{10} P\right)$ and statistically significant scores $\left(P \leq 10^{-5}\right)$ are shown with a light-orange to deep red color scale, see legend; non-significant scores are shown in white. MRinverter scores are based on MR activity inversion analysis in drug- vs. vehicle control-treated DU145 cells. For visualization purposes, heatmap results were clustered by hierarchical clustering. One among many statistically significant PGD-Loops is highlighted as an example. B. Highlighted PGD-Loop:_Circos plot showing the PGD-loop highlighted in panel A, comprising a SU2C patient (SC_9182_T), his cognate GEMM-DT (CMZ315), and the drug trametinib, and associated integrated $P$-value. C. GSEA analysis: GSEA plots showing the (a) patient to cognate GEMM-DT fidelity analysis (left), (b) the MR-inversion score by trametinib, as assessed for the patient's MRs (middle), and (c) the MR-inversion score by trametinib, as assessed for the cognate GEMM-DT. 
Vasciaveo et al.

\section{Figure 6: Selection and validation of candidate drugs}

A,B. Drug prioritization: FDA approved drugs $(n=117)$ (rows) were prioritized as candidate MRinverters of either patients from the SU2C cohort $(n=212)$ (columns in Panel A) or GEMM-DTs $(n=91)$ (columns in Panel B), using drug perturbation data from DU145 cells. Drugs were filtered based on screened concentration ( $\leq 1 \mathrm{uM}$ ) and patient coverage, i.e., only those identified as a MR-inverters for $>50 \%$ of the human samples were considered. Relevant phenotypic variables for either patients or GEMM-DTs are shown in bars at the top each heatmap. The MR-inverter score $\left(-\log _{10} P\right.$, as computed by aREA) is shown using a white $\left(P>10^{-5}\right)$ to dark blue heatmap (see legend), see STAR Methods. The blue barplots on the right summarize the number of patients or GEMM-DT predicted as sensitive to each drug. Black arrows to their right point to candidate drugs selected for validation, while the grey arrows point to cabazitaxel, the standardof-care for mCRPC, which was significant in the patient but not in the GEMM-DT analysis. In panel $\mathrm{B}$, the yellow barplot at the top shows the number of drugs identified as significant MRinverters for each GEMM-DT.

C-F. Preclinical validation. Selected drugs were validated in vivo, in allograft models derived from the cognate GEMM-DT CMZ315 (see Figure 5). Allografts were grown subcutaneously in nude mouse hosts and treated with predicted drugs, vehicle control, and negative control (cabazitaxel) for the times indicated, see STAR Methods. C. Summary of tumor volume changes over the treatment period. D. Summary of tumor weights, following sacrifice. $P$-values for $C$ and $D$ were computed by one-way ANOVA at the last time point, compared to Vehicle treated tumors and adjusted for multiple hypothesis testing with Dunnett's test (10 animals were enrolled to the vehicle control arm and 5 animals were enrolled on all drug treatment arms). E. Representative images of final tumor sizes in vehicle control and negative control-treated allografts. F. Representative images of final tumor sizes in allografts treated with predicted drugs. G. 
Vasciaveo et al.

Pharmacodynamic assessment of drug-mediated MR-inversion by GSEA analysis for the four predicted drugs.

\section{Figure 7: Co-clinical validation of candidate drugs}

A, B. OncoLoop analysis of patient derived xenograft (PDX) models: A. Similar to Figure 5A we show three heatmaps representing the fidelity and MR-inverter scores for 6 LuCaP PDX tumors (columns in left heatmaps), 5 GEMM-DTs, and 28 drugs. See Table $\$ 7$ for the MR-inverter score of all drugs as assessed for each of the PDX models. A specific PGD-Loop, comprising a PDX (MC005/LuCaP73), its cognate GEMM-DT (CMZ315), and two of the top-predicted drug candidates tested in the allograft models (panobinostat and trametinib). For visualization purposes, heatmaps were clustered as in Figure 5A. B. GSEA analysis showing the fidelity and MR-inverter P-value for the MC005/LuCaP73 model.

C-E. Validation of predicted drugs in the PDX model. The MC005/LuCaP73 PDX was grown in a nude mouse hosts and treated with predicted drugs and vehicle control for the times indicated. C. Summary of changes in tumor volume over the treatment period. D. Summary of tumor weights following sacrifice. $P$-values for $\mathrm{C}$ and $\mathrm{D}$ were computed by one-way ANOVA at the last time point, compared to vehicle control-treated models and adjusted for multiple hypothesis testing with Dunnett's test. E. Representative images of final tumor sizes.

F. Pharmacotype analysis-i.e., identification of patient subsets predicted to elicit sensitivity to the same drug subsets, by cluster analysis-identified four subtypes, including patients most sensitivity temsirolimus, trametinib, panobinostat, as well as patient for which none of the three validated drugs was significant. For each patient, the score of the most statistically significant MRinverter drug is shown using a white (non-significant) to dark-blue color map, see legend; the second most significant drug is shown using a white (non-significant) to dark-red color map, see legend. This heatmap provides the rationale for a possible umbrella or combination trial where 
each patient (column) could be randomized to the most statistically significant MR-inverter drug (rows) validated in the preclinical study, based on its MR-inversion score, or to the combination of the two most significant drugs. The barplots to the right show the total number of patients predicted to be sensitive to each drug, as either most significant (blue) or second best selection (red). 
bioRxiv preprint doi: https://doi.org/10.1101/2022.02.11.479456; this version posted February 14, 2022. The copyright holder for this preprint (which was not certified by peer review) is the author/funder. All rights reserved. No reuse allowed without permission.

Vasciaveo et al.

\section{Figure 1}

\section{A. OncoLoop conceptual framework}

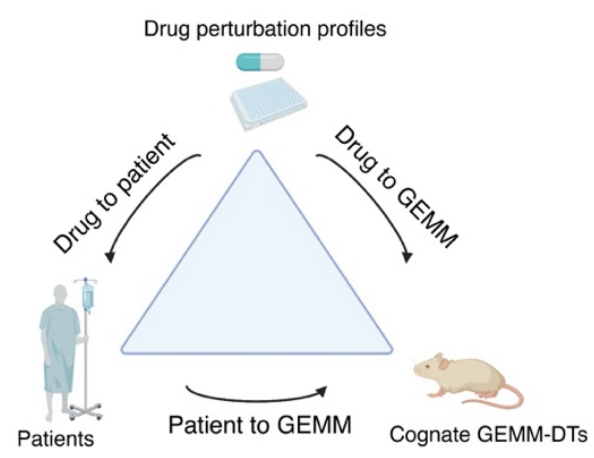

\section{B. Regulatory network analysis}

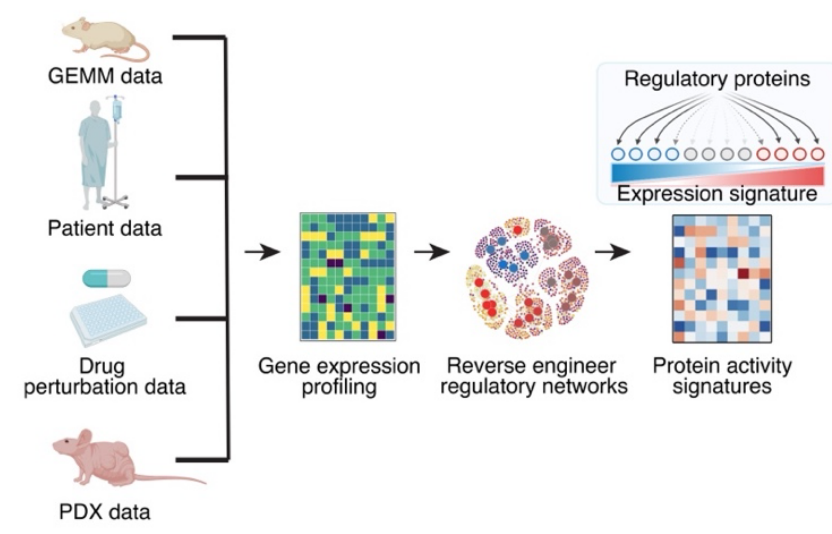

\section{OncoLoop analysis}
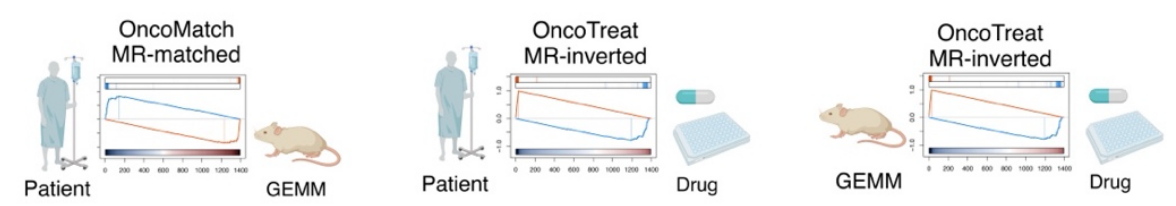

D. Drug prediction and validation

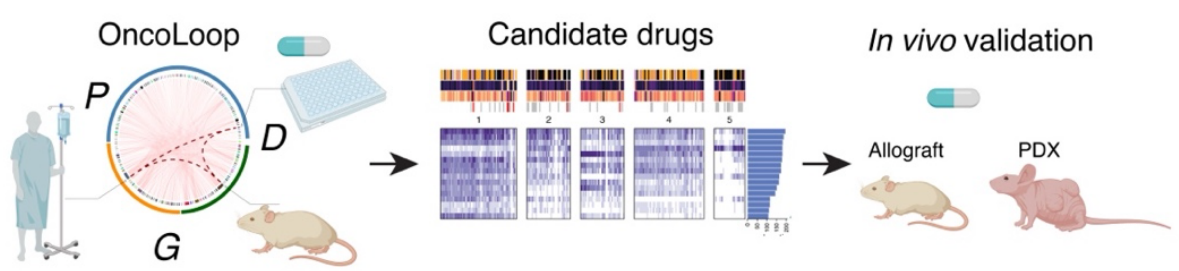


bioRxiv preprint doi: https://doi.org/10.1101/2022.02.11.479456; this version posted February 14,2022 . The copyright holder for this preprint (which was not certified by peer review) is the author/funder. All rights reserved. No reuse allowed without permission.

Vasciaveo et al.

Figure 2

A

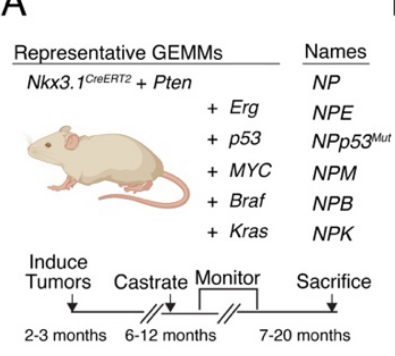

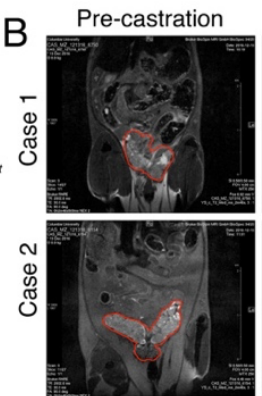
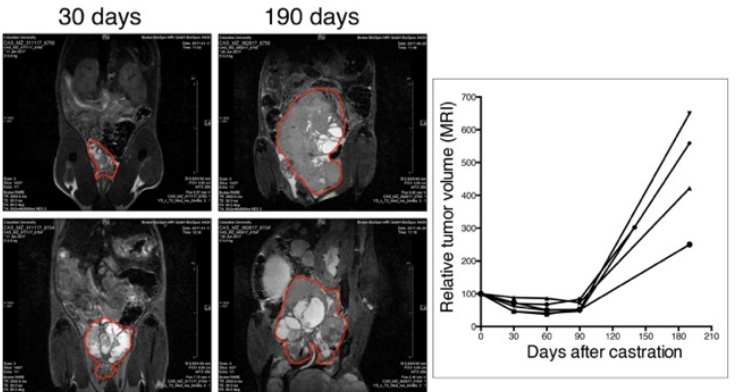

C

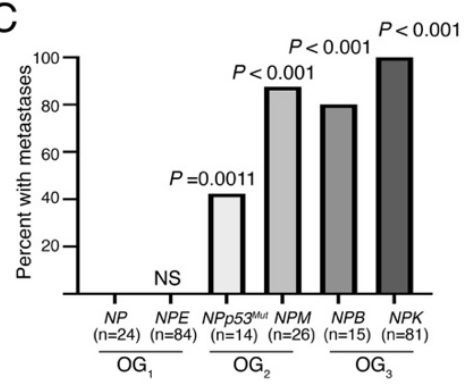

D

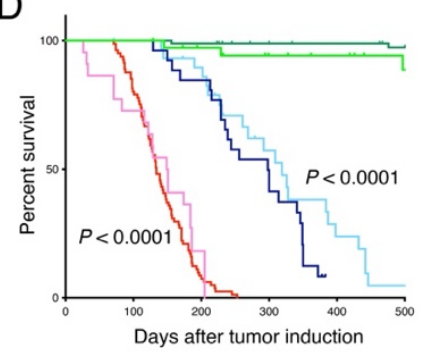

Outcome group $1\left(\mathrm{OG}_{1}\right)$

$-N P(n=37)$

Outcome group $2\left(\mathrm{OG}_{2}\right)$

$-\operatorname{NPM}(\mathrm{n}=30)$

$-\operatorname{NPp53}^{\text {mut }}(\mathrm{n}=26)$

Outcome group $3\left(\mathrm{OG}_{3}\right)$

NPB $(\mathrm{n}=22)$

$-\operatorname{NPK}(\mathrm{n}=81)$

E
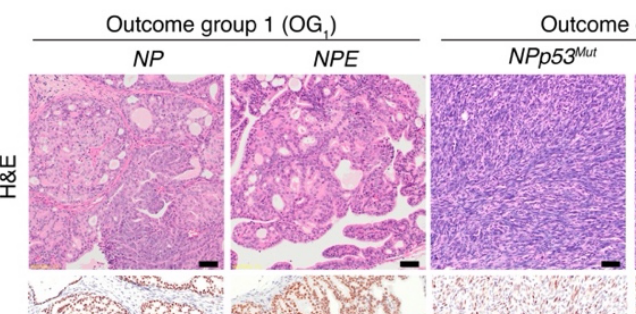

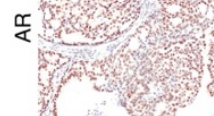

产
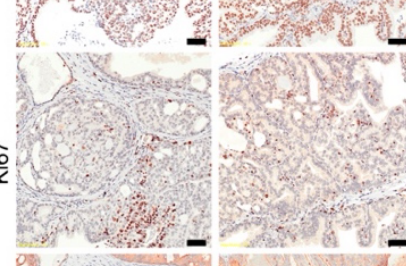

ํ.
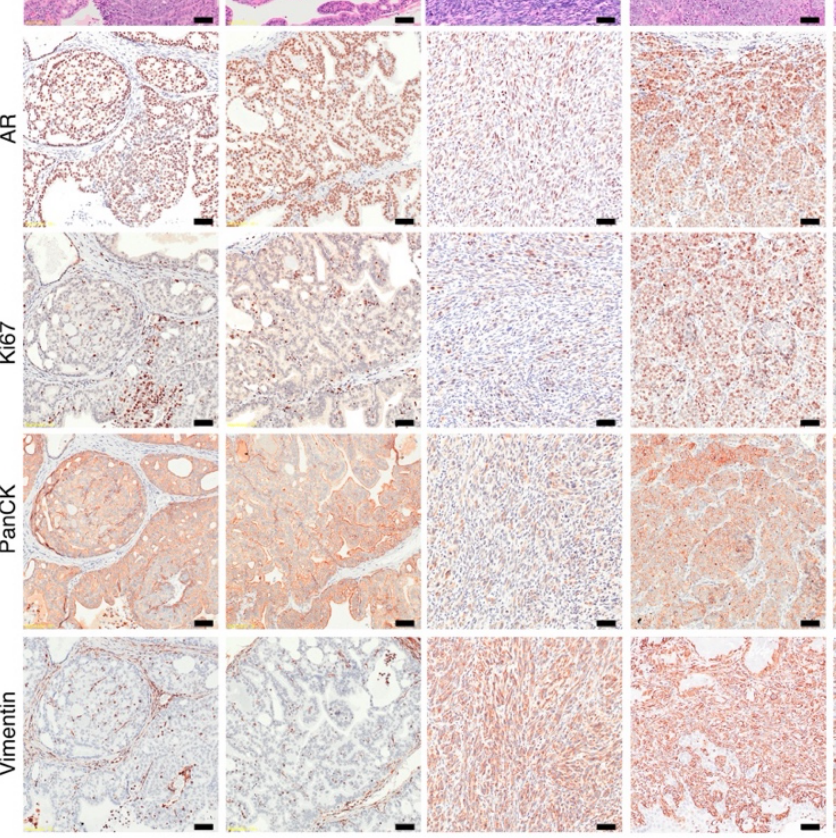

Outcome group $3\left(\mathrm{OG}_{3}\right)$

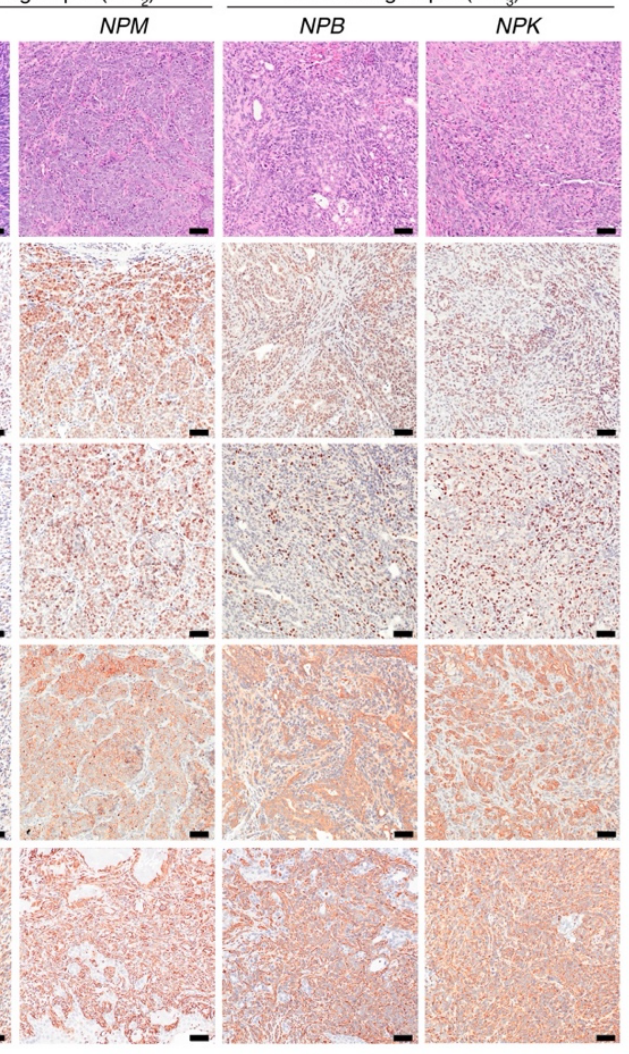


bioRxiv preprint doi: https://doi.org/10.1101/2022.02.11.479456; this version posted February 14, 2022. The copyright holder for this preprint (which was not certified by peer review) is the author/funder. All rights reserved. No reuse allowed without permission.

Vasciaveo et al.

Figure 3

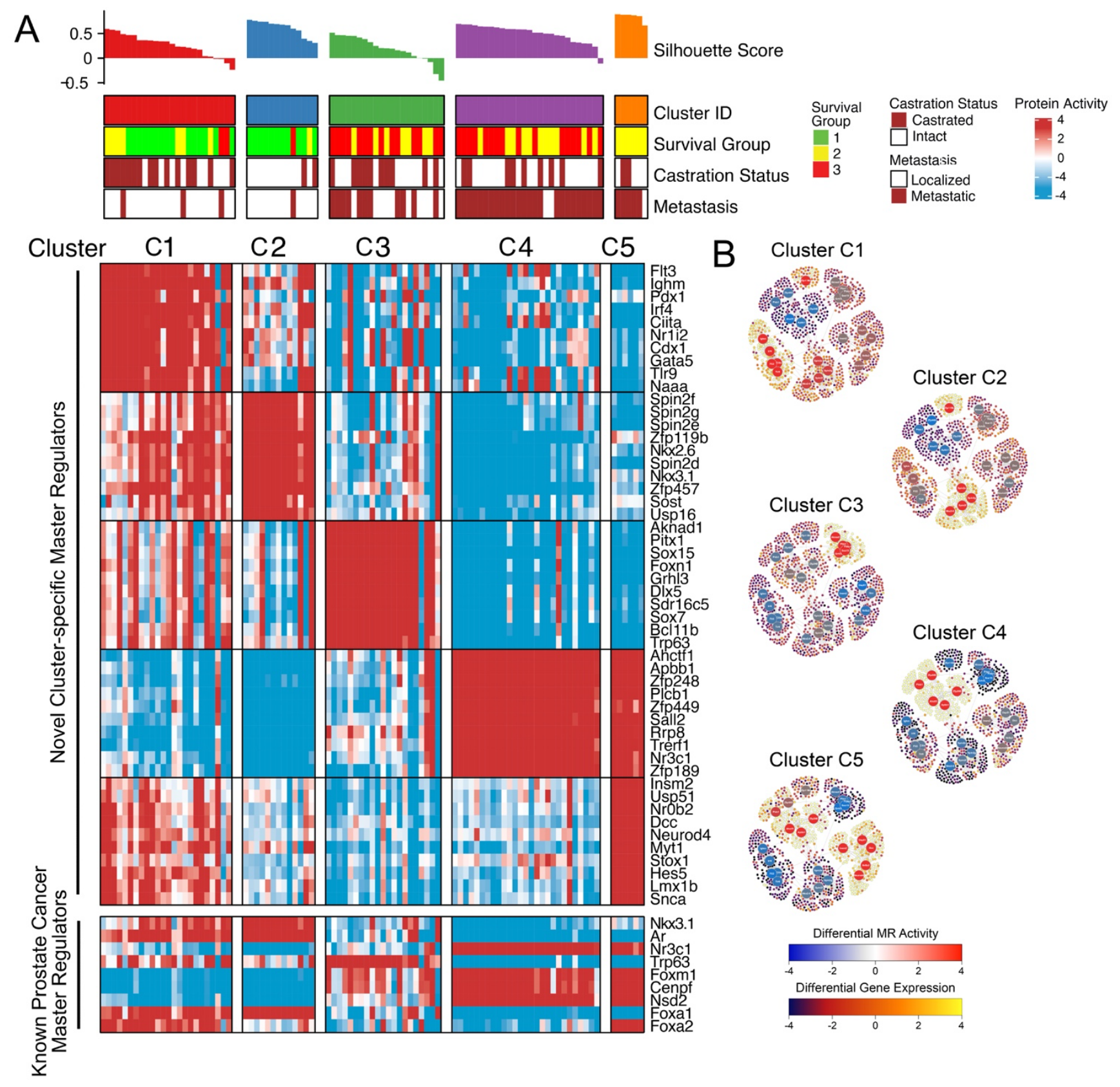


bioRxiv preprint doi: https://doi.org/10.1101/2022.02.11.479456; this version posted February 14, 2022. The copyright holder for this preprint (which was not certified by peer review) is the author/funder. All rights reserved. No reuse allowed without permission.

Vasciaveo et al.

\section{Figure 4}

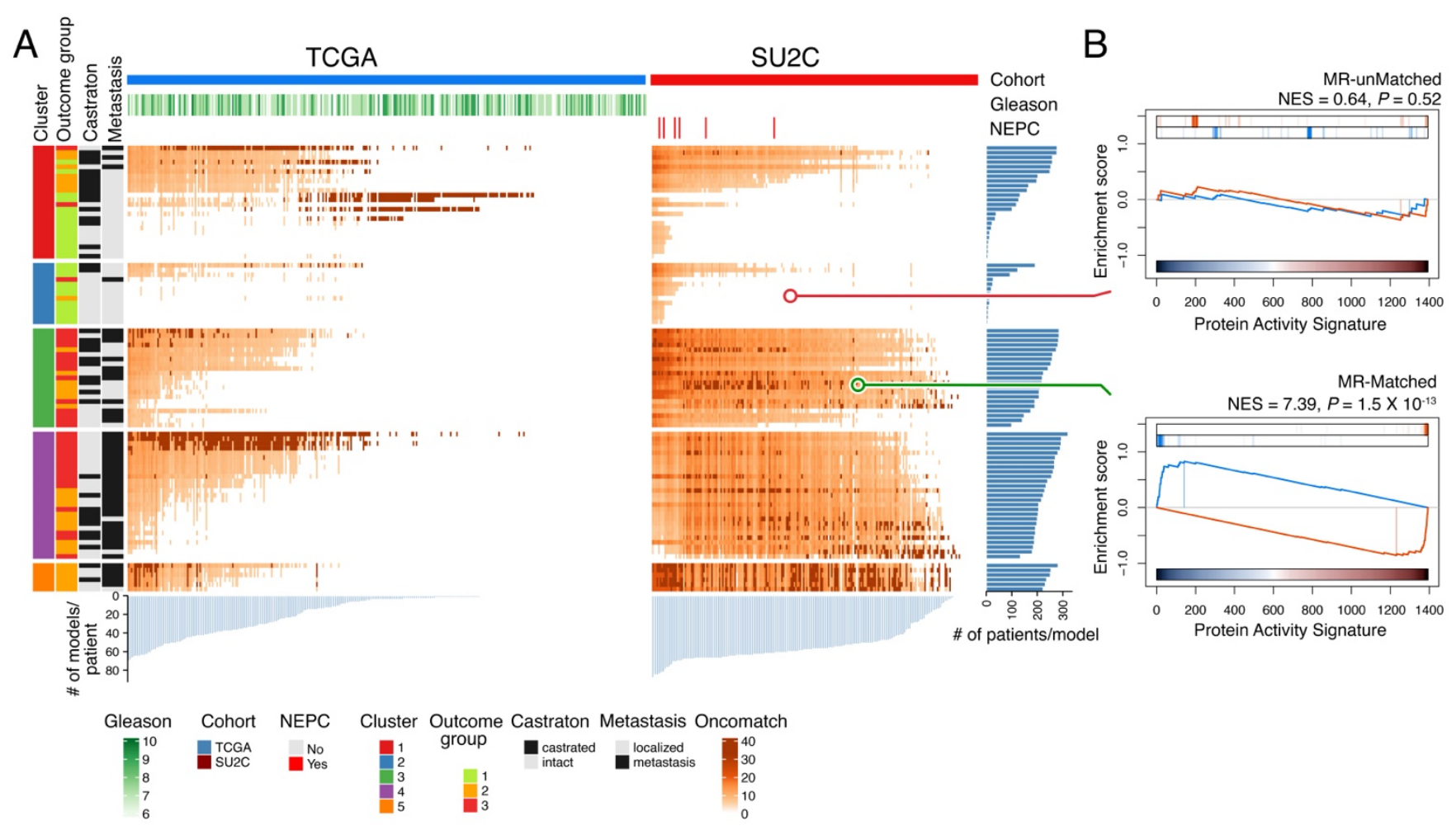


bioRxiv preprint doi: https://doi.org/10.1101/2022.02.11.479456; this version posted February 14,2022 . The copyright holder for this preprint (which was not certified by peer review) is the author/funder. All rights reserved. No reuse allowed without permission.

Vasciaveo et al.

\section{Figure 5}

A OncoMatch: Patient vs GEMM-DT

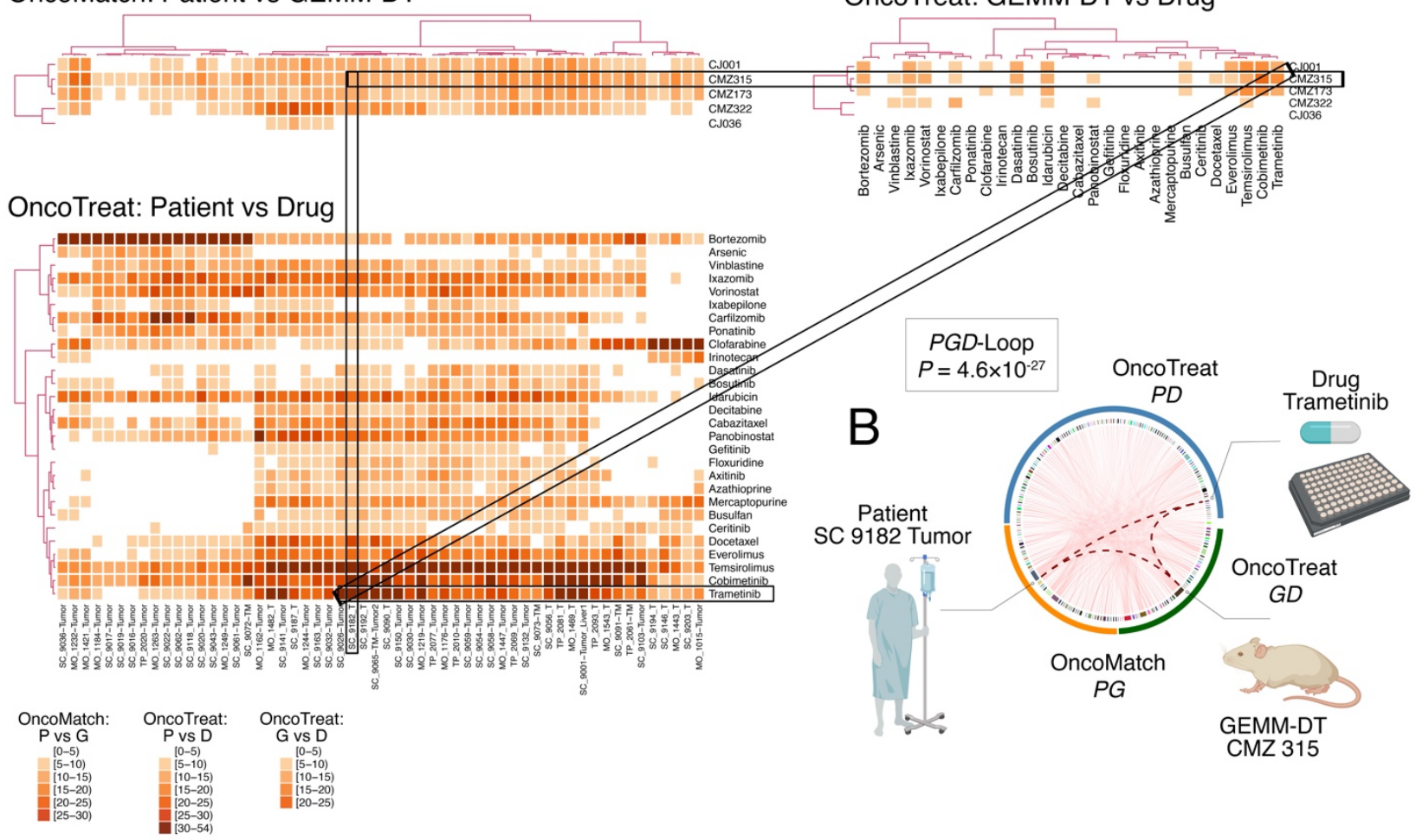

C

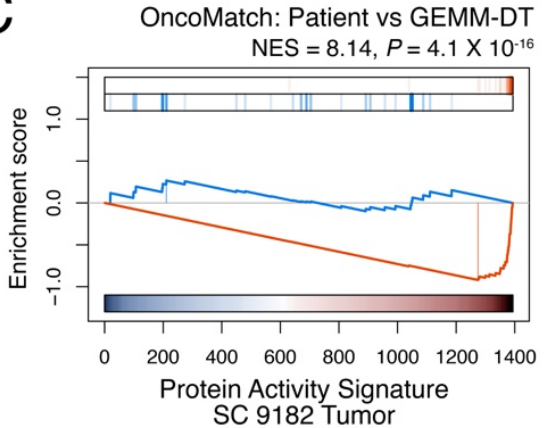

SC 9182 Tumor
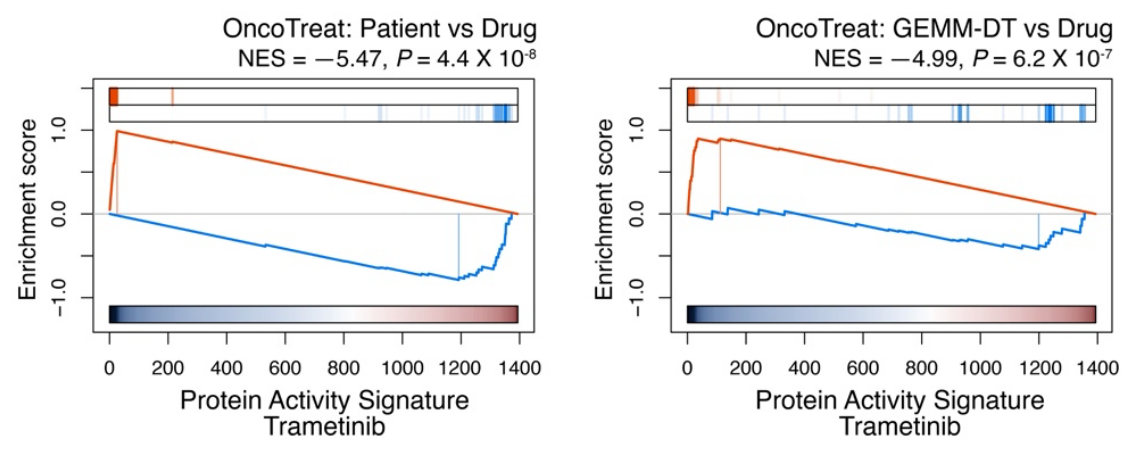
bioRxiv preprint doi: https://doi.org/10.1101/2022.02.11.479456; this version posted February 14, 2022. The copyright holder for this preprint (which was not certified by peer review) is the author/funder. All rights reserved. No reuse allowed without permission.

Vasciaveo et al.

Figure 6

A
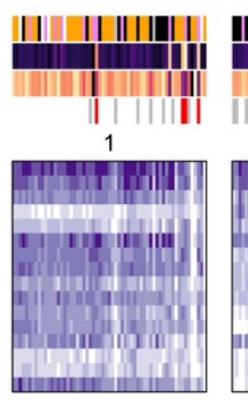

B

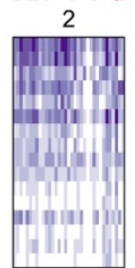

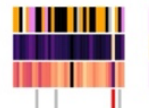
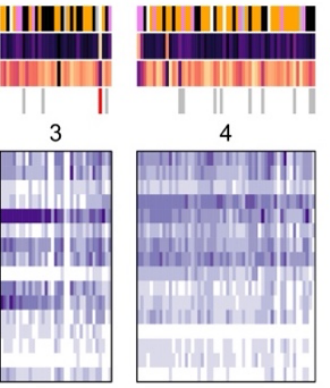

\|\|\|\|$_{\text {AR Score }}^{\text {NEPC Score }}$
1II NEPC

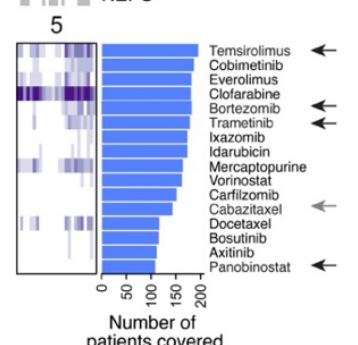

patients covered
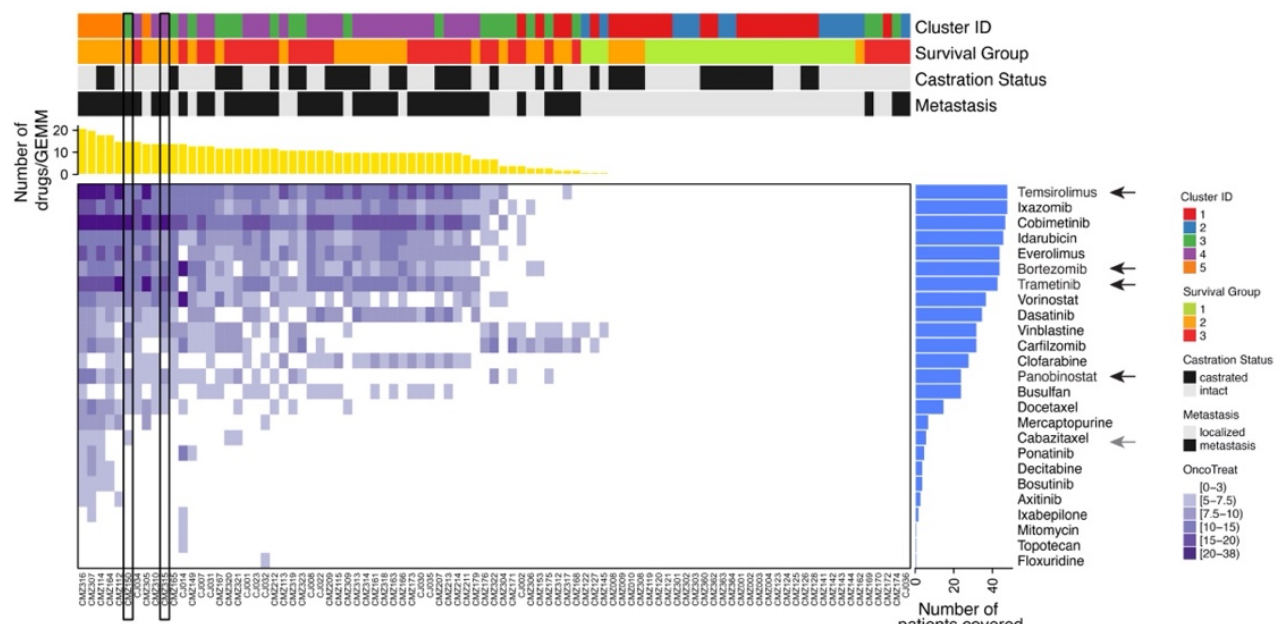

C

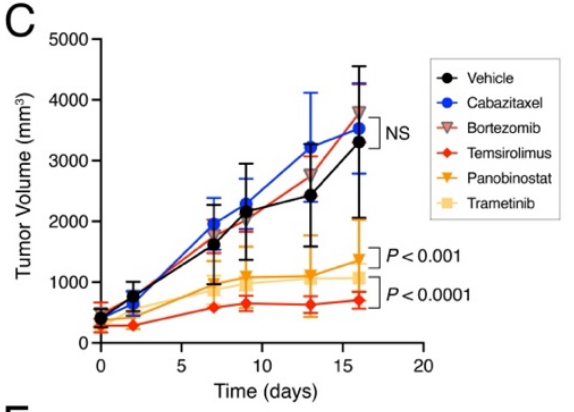

$\mathrm{F}$

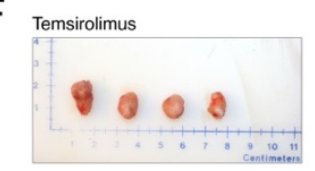

G Temsirolimus
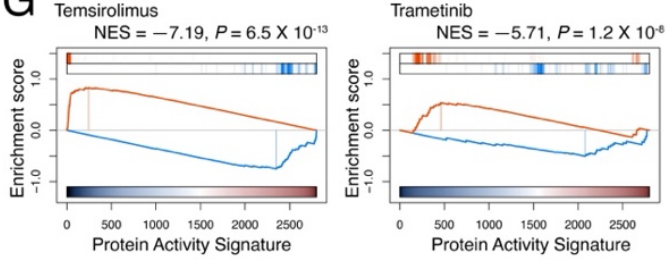

D

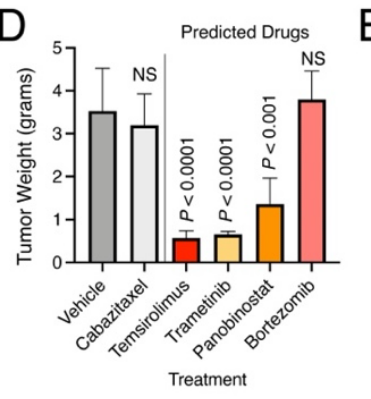

Panobinostat

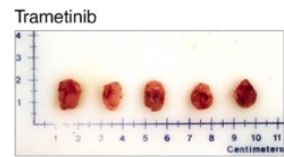

Panobinostat

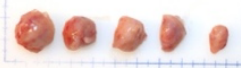

Panobinostat

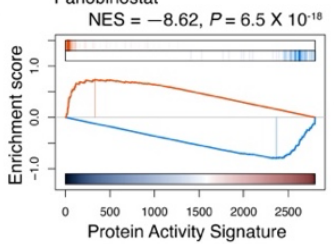

E
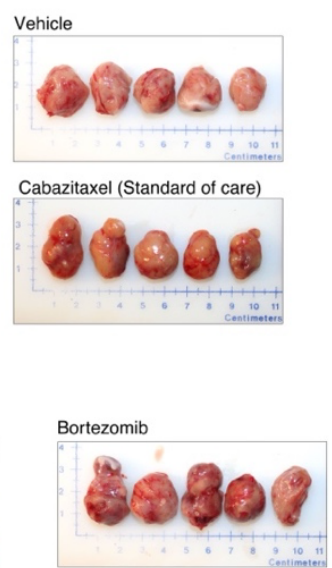

Bortezomib

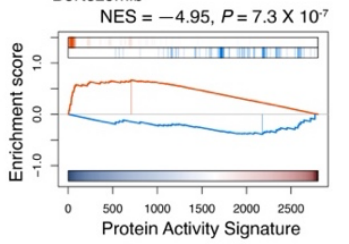


bioRxiv preprint doi: https://doi.org/10.1101/2022.02.11.479456; this version posted February 14,2022 . The copyright holder for this preprint (which was not certified by peer review) is the author/funder. All rights reserved. No reuse allowed without permission.

Vasciaveo et al.

Figure 7

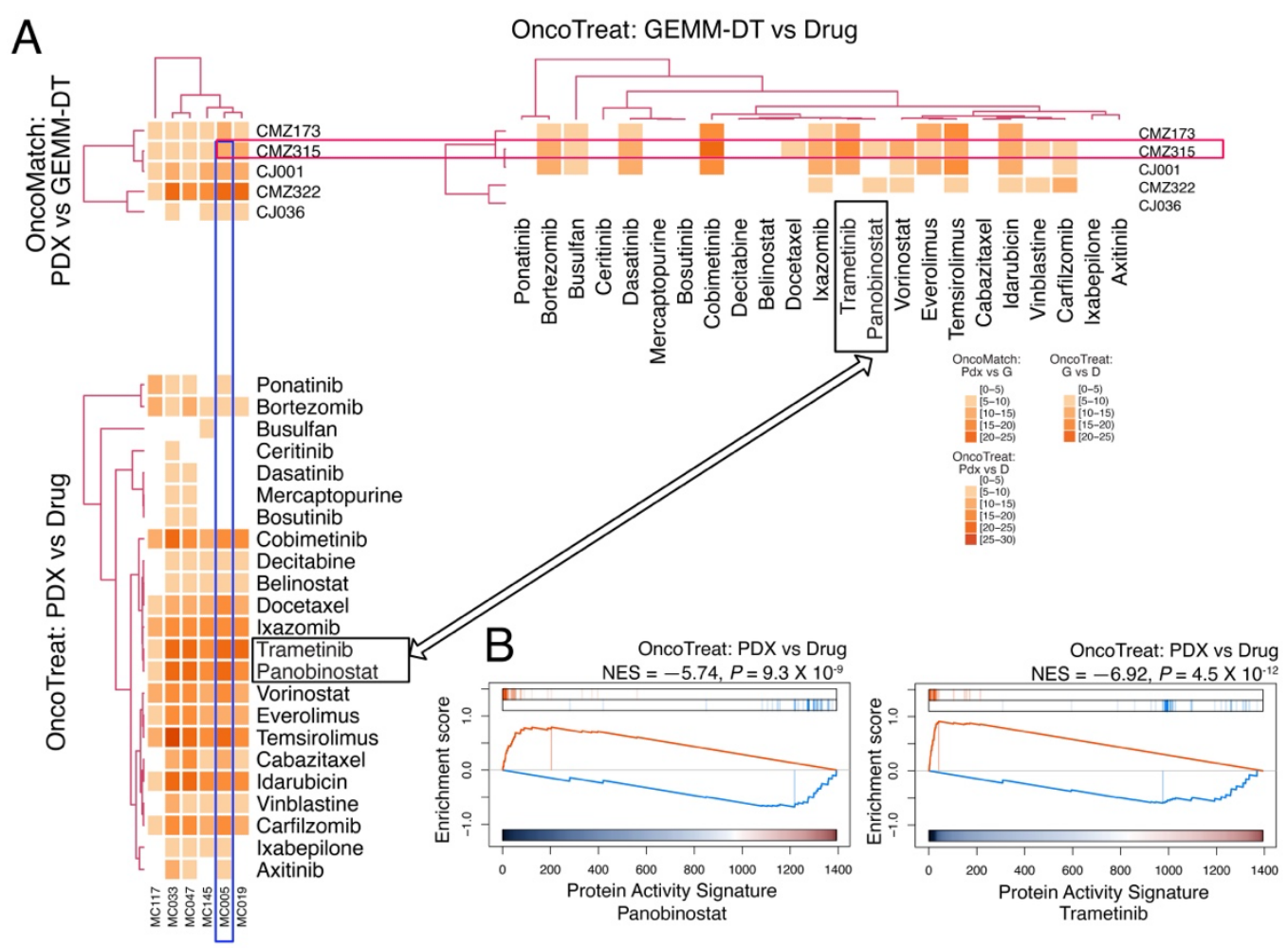

C

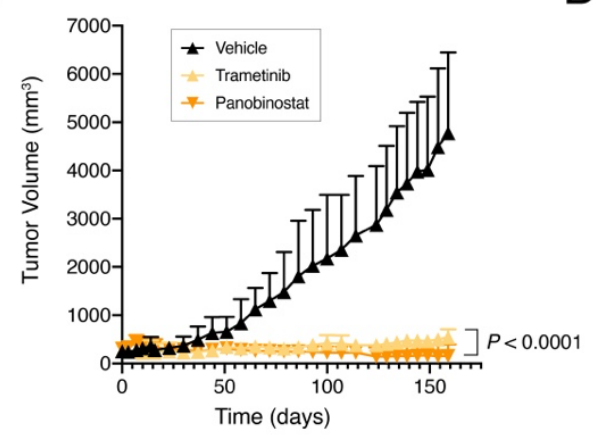

F
E

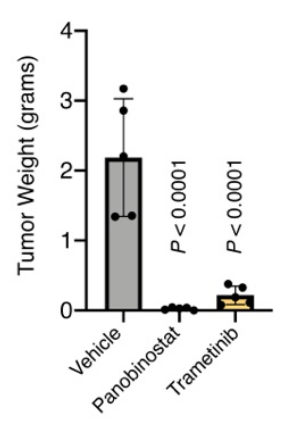

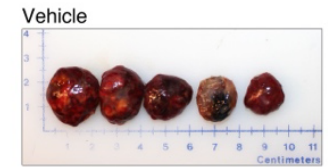

Panobinostat

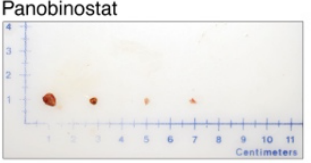

Trametinib

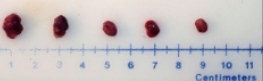

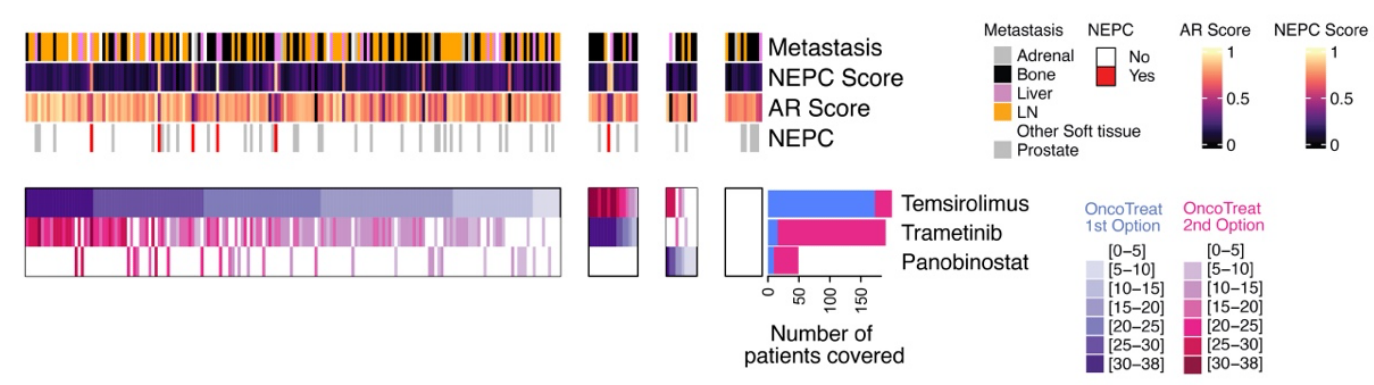

